\title{
Theories of the deep: combining salience and network analyses to produce mental model visualizations of a coastal British Columbia food web
}

\author{
Jordan Levine $^{1}$, Michael Muthukrishna $^{2,3}, \underline{\text { Kai M. A. Chan }}^{1}$ and Terre Satterfield ${ }^{1}$
}

\begin{abstract}
Arriving at shared mental models among multiple stakeholder groups can be crucial for successful management of contested social-ecological systems (SES). Academia can help by first eliciting stakeholders' initial, often tacit, beliefs about a SES, and representing them in useful ways. We demonstrate a new recombination of techniques for this purpose, focusing specifically on tacit beliefs about food webs. Our approach combines freelisting and sorting techniques, salience analysis, and ultimately network analysis, to produce accessible visualizations of aggregate mental models that can then be used to facilitate discussion or generate further hypotheses about cognitive drivers of conflict. The case study we draw upon to demonstrate this technique is Clayoquot Sound UNESCO Biosphere Reserve, on the west coast of British Columbia, Canada. There, an immanent upsurge in the sea otter (Enhydra lutris) population, which competes with humans for shellfish, has produced tension among government managers, and both First Nations and non-First Nations residents. Our approach helps explain this tension by visually highlighting which trophic relationships appear most cognitively salient among the lay public. We also include speculative representations of models held by managers, and pairs of contrasting demographic subgroups, to further demonstrate potential uses of the method.
\end{abstract}

Key Words: British Columbia; food webs; mental models; network analysis; salience analysis

\section{INTRODUCTION}

Considerable work has been done describing the potential for mental models research to enrich our understanding of complex social-ecological systems (Lynam and Brown 2011). In theory, such insights about how people make sense of complex systems can help improve multistakeholder management of shared resources. Specifically, studies in organizational management have found that it is precisely the development of shared mental models among multiple parties that serves as the lynchpin for successful negotiations (Brodt and Dietz 1999, Liu 2004).

However, actively constructing shared mental models among different parties in a resource conflict or management context is not necessarily straightforward. One challenge is that people's innate beliefs about their social-ecological system are often tacit, and thus cannot be stated directly without adequate elicitation efforts (Jansen et al. 2006, Beratan 2007). Another is that, in many resource conflict or multistakeholder management contexts, actors constitute not only individuals, with individual mental models, but also loosely affiliated groups, or blocs, more usefully described to each be acting within the logic of a more nebulous, "aggregate" mental model. These group-level aggregate models (sometimes called "cultural models"; see Appendix 1) can prove uniquely challenging to elicit, let alone represent with meaningful validity.

In this paper we demonstrate a first iteration of an approach synthesized specifically to elicit and represent widespread tacit beliefs about a regional food web. In particular, we outline how it is possible to combine freelisting tasks, salience analysis (Smith and Borgatti 1997), and network analysis (Gephi 2014) to ultimately create tangible, empirically responsible visual representations of aggregate mental models that are unique in highlighting the effects of cognitive constraints on people's thinking.
We report on our application of these techniques to one specific case study site: the Clayoquot Sound UNESCO Biosphere Reserve, on the west coast of Vancouver Island (see Appendix 2). There, a resurgence of the once extirpated sea otter (Enhydra lutris), combined with a regional decline in fisheries, had, at the time of our fieldwork in 2012, created an atmosphere of tension among locals and government officials over how best to manage marine resources in rapid flux. In the context of in-depth semistructured interviews, First Nations, in particular, had been lamenting the voraciousness of the sea otter, which is protected by law but perceived to be decimating edible shellfish populations once a staple of First Nations' diets. Non-First Nations' sentiments toward the otter were often more positive, with some welcoming the creatures for their endearing appearance, and potential ecotourism value. Nonetheless, there was considerable ambivalence among local residents as a whole regarding the sea otter's dramatic recent resurgence. In stark contrast to the lay public, government managers were highly enthusiastic about otters' return to the region, specifically citing positive knock-on effects (a "trophic cascade") whereby otters' reduction of the sea urchin population could lead to denser kelp beds, and hence more habitat for a wider range of biodiversity.

For the sake of methodological clarity, in this paper we focus mainly on analyzing the data elicited from our interviews with lay public participants as a whole. In so doing, we derive a visual model that reveals which trophic relationships are tacitly structuring popular conceptions of the local marine food web. Before concluding, we also present a series of models derived both from government manager interview data, and by dividing the lay public data into two pairs of contrasting demographic subgroups, First Nations vs. non-First Nations, males vs. females. Because of logistical constraints, these latter models are speculative, but demonstrate how the method, if refined, could be used to generate

${ }^{1}$ Institute for Resources, Environment and Sustainability, University of British Columbia, ${ }^{2}$ Department of Human Evolutionary Biology, Harvard University, ${ }^{3}$ Department of Social Psychology, London School of Economics 
hypotheses regarding otherwise tacit cognitive drivers of management conflict between groups.

\section{METHODS}

Our method consisted of three phases: (1) in-depth structured interviews in which freelisting and related tasks were used to elicit participants' beliefs about the local social-ecological system, and specifically their individual mental models of the local marine food web; (2) salience analyses of the collective freelist data at the group level using the software package ANTHROPAC (Borgatti 1996); and finally (3) network analyses of the group-level food web salience data using the software package Gephi (2014). Ultimately, we used Gephi to render these results visually, as food web models that highlight which species, and which trophic connections among which species, are most cognitively salient to participants (see Figs. 1 to 6).

\section{Interviews}

Over the course of several months in the spring of 2012, we completed in-depth structured interviews with 67 local residents of Clayoquot Sound, as well as with four key government resource managers responsible for conservation prerogatives in the region. Local residents were recruited primarily using popular community poster boards in the area, supplemented by word-ofmouth snowball sampling. For those participants who were interviewed on recognized First Nations-administered lands, contact was first established with elected community leaders who subsequently introduced us to individual community members, which was also followed by word-of-mouth snowball sampling. Government managers, as a separate sample, were identified and approached based on prior working relationships and job title.

The 67 local-resident participants ranged in age from 20 to 80 . Within this group, there were 29 females, and 38 males. Of these participants 41 self-identified as non-First Nations, while the remaining 26 were self-identified First Nations, primarily from the Ahousaht and Toquaht nations, with a few participants from the Tla-oh-qui-aht and Yuułu?ił?ath (Ucluelet) nations. Participants from the latter two bands granted interviews outside of their communities, in Tofino and Ucluelet proper, while interviews with the former were conducted on tribally administered lands. All participants were offered financial compensation for their time at a rate of CAD $\$ 15$ per hour. The study was deemed low-risk, and subsequently approved, by the University of British Columbia Research Ethics Board as per institutional regulations.

The interviews consisted of a relatively lengthy structured protocol, conducted at a place of the participants' choosing. The protocol involved a range of freelisting tasks, sorting tasks, and drawing tasks, as well as number of questions pertaining to participants' demographics, their beliefs about the local ecology, and their otter-management preferences (see Appendix 3 for the full protocol).

The relevant segment of the interview for the purposes of this paper began with a freelisting task, wherein the participants were asked to verbally list "as many species and/or resources [you] can think of on the west coast of Vancouver Island." As participants did so, they were requested to write the name of each species or resource down on small individual cards in the order that they came to mind. This ordering ultimately gave us a measurement of species' relative "cognitive accessibility", to participants, i.e., how readily a given species comes to a given participant's mind, and, by inference, a sense of which species feature most prominently in participants' mental models. We later conducted a salience analysis (Smith and Borgatti 1997) on this ordering data to explore species' relative accessibility at the group level (see Appendix 4, Tables A4.1 to A4.6, for a relevant selection of this data).

Subsequent to the first freelisting task, the protocol aimed to elicit participants' beliefs specifically regarding trophic interactions among species. To do this, we devised a task wherein participants were provided with a box full of a large volume of wooden popsicle sticks. On each popsicle stick we had written the word "eats" with a long arrow pointing in one of two directions. The interviewer then requested participants to begin connecting the pieces of paper on which they had written species' names by using the popsicle sticks to show "what eats what" in the local ecosystem.

This technique is similar to the "diagram method" of mental model elicitation (see Green et al. 2003), but with a few consequential innovations. First is the relatively tangible nature of the exercise we implemented, whereby participants were free to physically manipulate "species" and interconnections with their hands, in space, to help them work through their implicit knowledge.

Next, participants were asked to verbalize their thinking as much as possible during this task, so the interviewer could clearly understand their reasoning. Crucially, as they did this, the interviewer recorded the order in which the participants thought of, and represented, the various trophic interactions. This preservation of ordering data is what enabled later salience analysis, and is thus a key feature of the elicitation method.

The participants' task ended once they had connected as many species, one to the other, as possible. For each given participant, the end result was thus a visually tangible depiction of his or her mental model of the local food web, which was then photographed (see Appendix 5, Fig. 5A.1, for an example).

\section{Salience analysis}

Once all the species orderings and trophic web data had been collected, we began to analyze it at the aggregate level. Understanding this shift from eliciting data at the individual level, to analyzing it in the aggregate, is crucial because this is what allows inferences to be made regarding average salience.

With the aggregate level in mind, we first analyzed the freelisted species data using the salience analysis function of ANTHROPAC (Borgatti 1996). Salience analysis helped us approximate which species were most readily recalled by people, thus providing one measure of tacit cognitive tendencies that can affect reasoning about the local ecosystem (see Appendix 4, Tables 4A.1 to 4A.6 for relevant selections from these initial results).

To do this, we used ANTHROPAC to calculate a Smith's salience index (Smith and Borgatti 1997), or simply "salience score" for each species mentioned by participants. Calculating salience indices is a cognitive anthropological technique for analyzing interviewee-generated lists of terms, which has since been adopted across multiple disciplines (Smith and Borgatti 1997, Sutrop 2001, Barg et al. 2006, Thompson and Juan 2006, de Morais 2009, 
Pradhan and Ram 2010, Ghorbani et al. 2011, Malan and Neuba 2011, Dongre and Deshmukh 2012, Gravlee et al. 2013). A given term's Smith's salience index $(S)$ among a group of participants is a function of both the frequency with which the term is mentioned during a freelisting exercise, i.e., the number of participants who include a given term on their respective lists, as well as a function of the term's average position on participants' lists. In our case, terms' positions on the list were equivalent to the order in which those terms were mentioned by participants. Terms that are mentioned relatively often and are positioned relatively highly on people's lists, i.e., mentioned early on, obtain the highest saliency scores, and vice versa (Barg et al. 2006). Scores range from 1 (highest) to 0 (lowest; see Appendix 4 for a full description of the calculation).

We then proceeded with a methodological innovation, in which we took participants' lists of trophic interactions, preserving the order in which participants mentioned each interaction (a key step), and treated these orderings as freelists in and of themselves. The first trophic interaction a participant listed was taken to be the most cognitively salient, i.e., most readily thought of, for that participant, the second, the second-most salient, and so forth. Group-level analyses of this data then provided a general sense of which interspecies relationships were, on average, relatively cognitively prominent for participants at the aggregate level. In other words, for each demographic subgroup, the salience analysis produced an ordered list of "what eats what," indicating by salience index which trophic relationships, e.g., otters eating urchins, or killer whales eating salmon, were most readily recalled by people, suggesting which ecological relationships most directly structure people's mental models of the system. (See Appendix 6).

The second crucial feature of our synthesized method is that, because this data specifically characterizes perceived relationships between species, it can also then be further analyzed as a network of species connected by trophic relationships, with each connection weighted by salience index (see Appendix 6, Tables 6A.1 to 6A.6 for relevant sample data). This second innovation is what ultimately enabled us to produce a uniquely data-rich visualization of local residents' aggregate mental model of the Clayoquot food web (see Fig. 1), the primary methodological contribution of this paper.

To test the hypothesis-generating capacity of the method, we also twice experimented with dividing the local-resident sample into preidentified (see Appendix 7) contrasting halves: once into First Nations versus non-First Nations, and once into males versus females. The results thereof are purely demonstrative, not statistically derived, and so are listed under "Speculation" (see Figs. 3-6), alongside a similarly demonstrative model derived from the responses of our small $n$ (4) of government managers (see Fig. 2).

\section{Network analysis}

Having used ANTHROPAC's salience analysis function to produce lists of trophic relationships with salience indices (see above for a description, and Appendix 6 for a sample of the data), we then took that data and entered it into the free network analysis software package Gephi (2014).

Once this data had been entered into Gephi, we could then proceed with a range of network analyses that calculated a number of
Fig. 1. Aggregate mental model of the regional food web: local residents. The size of a species' name in a given group's aggregate mental model corresponds to that species' out-degree centrality, the size of a species' circle corresponds to its indegree centrality, and the darkness of a species' circle corresponds to its betweenness centrality. Finally, the width of an arrow corresponds to that given trophic relationship's average cognitive accessibility. From this visualization, it thus appears that local residents' average conception of the Clayoquot Sound food web revolves largely around the feeding habits of large charismatic megafauna (bears, killer whales, wolves, and cougars), as well as that of sea otters. Local residents appear aware of humans' role as a prolific predator in the system, but this role is far less salient, or immediately apparent, to them than the behavior of other predatory mammals. Salmon, as a taxonomic class, appear to occupy a uniquely central role in local residents' conceptions of the social-ecological system, both as a crucial "species" for energy transfer throughout the food web, as well as a "species" that is fed on (along with fish in general) by the widest diversity of predators.

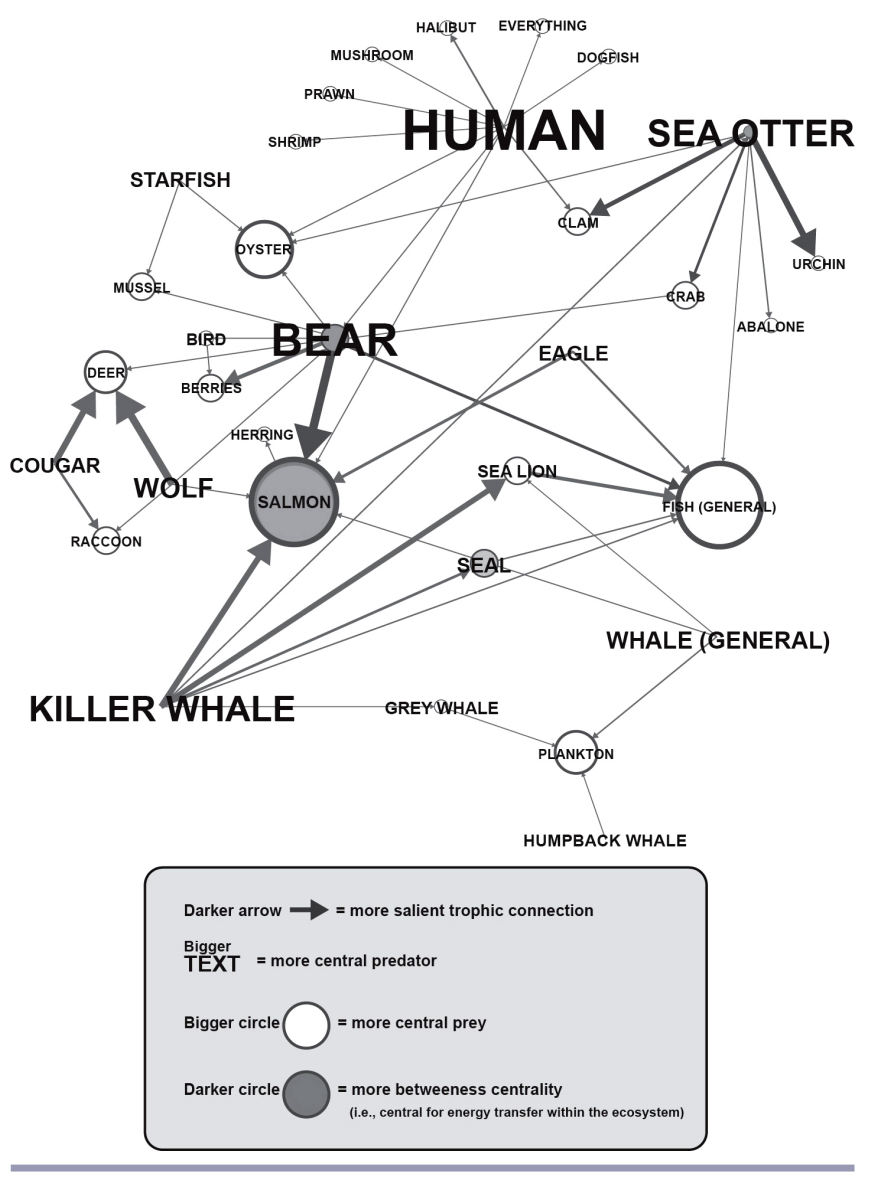

"network-centrality" measures for each species. Ultimately, this enabled us to produce data-rich visualizations of aggregate mental models of the local food web (see Figs. 1 to 6), depicting both which connections ("what eats what") appear to be most cognitively accessible to people, as well as which species feature 
as the most prominent predators, prey, or ecological connectors as determined by network-centrality measures.

\section{RESULTS}

We outline some of the most basic measures that network analysis allows us to calculate that are relevant to mental models of food webs. For clarity, we describe how these features are represented in our chosen visualization scheme. We then follow with a visual presentation of the results of the network analysis itself (i.e., Fig. 1).

\section{Network analysis of imagined food web data: measuring and visualizing centralities}

Network analyses consist of two core components: nodes and edges. Nodes simply constitute the objects or "things" that are related to one another in some way. In the case of our Clayoquot Sound food web data, these nodes are the plants and animals that participants mentioned during their trophic connection task (see Appendix 5). Edges are the connections between two nodes. Edges can vary in a number of ways, including by their relative strength (in our case, their "weighting," as determined by salience index), and their directionality (in our case, the direction of predation).

There is a plethora of ways to measure the relative importance of nodes to a network, i.e., in our study, the importance of species to the wider food web. Such metrics are referred to in the network analysis literature as "centrality measures." Given that our present study comprises merely a first iteration of a new technique, for simplicity's sake, we have chosen to highlight only three of the most basic and seemingly relevant centrality measures in our visualizations: "in-degree" centrality, "out-degree" centrality, and so-called "betweenness" centrality.

\section{Out-degree centrality: bigger text depicts a more voracious predator}

We chose the size of each species' name to correspond to that species' relative out-degree centrality. In this case, out-degree centrality equates specifically to the number of other individually named plants or animals that participants believe a given species preys upon. Thus, the more diverse range of prey a given species is believed to have, the larger its name appears in the network, and vice versa.

\section{In-degree centrality: bigger circles depict more widely targeted prey}

The size of a species' circle, inversely, was selected to represent that species' relative in-degree centrality. In our study, this equates specifically to the number of individually named predators that participants believe prey on the given species. Thus, the greater the diversity of predators a species is believed to have, the bigger its corresponding circle appears in the network.

\section{Betweenness centrality: darker circles depict more crucial connectors}

We chose the darkness of a given circle to correspond to that species' perceived betweenness centrality in the food web. Betweenness centrality is one of the most basic measures of how pivotal a node is as a connector among other nodes in a network. Gephi calculates this measure based on an algorithm refined by Brandes (2001).

Specifically, analyzing for this measure of centrality identifies which nodes in a network are most frequently located along the most direct path between all other pairs of nodes in the network.
Fig. 2. Aggregate mental model of the regional food web: government managers. The feeding habits of sea otters, humans, and bears appear especially salient for government managers. Managers also appear to think of fish species and those species' respective trophic relationships at a much higher phylogenetic resolution than do civilians. Furthermore, unlike local civilians, managers appear particularly cognizant of the trophic relationships that constitute the kelp-and-urchin centered subsystem that is directly impacted by the trophic cascade of sea otter predation. Finally, the charismatic killer whale appears conspicuously absent from managers' cognitive representations of the marine ecosystem, while two other, less iconic predators, sharks and sunflower starfish, both serve important structural roles in managers' aggregate model. (Note that this visualization is demonstrative of the method's potential uses, not necessarily representative of a demographic with statistically distinct responses).

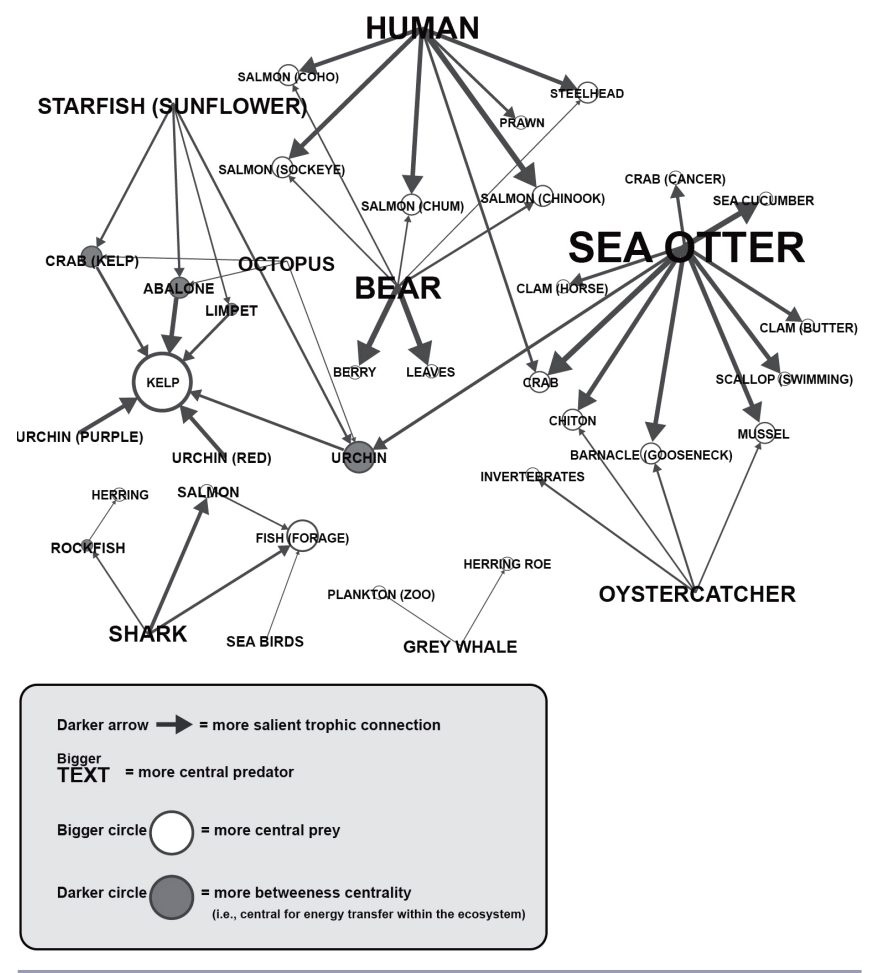

In the case of our food web data, this equates to how directly a given species functions as a connector in the energy transfer among all other species in the food web. To our knowledge, this is the first instance of such a use of betweenness centrality in the social-ecological mental models literature.

Edge weight: bigger arrows depict greater cognitive accessibility Finally, because our networks represent not an actual food web, but rather participants' aggregate cognitive representation of that food web, we also included in our visualizations the relative cognitive accessibility of each trophic interaction. This greatly increases the inferential relevance of each visualized aggregate mental model because it enables the viewer to immediately grasp which relationships between which species feature most prominently in which peoples' minds. This is the most crucial 
contribution of the method because it makes immediately accessible otherwise tacit patterns in participants' ecological cognition.

Seeing these weightings immediately allows the reader to infer which relationships among which species are likely to most actively structure people's thinking about the ecosystem. We chose to depict this relative cognitive accessibility of trophic relations using the size of the arrows that connect species in the network one to another. In other words, while the direction of each arrow represents the direction of predation, the size of each arrow represents the cognitive weight of that trophic connection for participants, as calculated by salience index. Thus, the more cognitively salient a given trophic connection is on average among a given group of people, the larger the arrow connecting the two relevant species in the visualization of that group's aggregate mental model.

For simplicity's sake, and to reduce visual clutter, we capped the number of trophic connections included in a given visualization. The metric we used as a threshold was once again the salience ranking we had calculated earlier using ANTHROPAC: only the 50 most salient trophic connections among species were included in this analysis for each of the demographic subgroups we studied. See Figure 1 for the visualized results of local residents' responses.

\section{DISCUSSION}

The relative size of the words in Figure 1 indicate that for the lay public in Clayoquot Sound, the most central predators, ranked in descending order by out-degree centrality (noted numerically in parentheses) include: human (10); bear (8); killer whale (6); sea otter (6); and wolf (4). It is these five species, in other words, that locals implicitly believe dominate the ecosystem. Conversely, the relative size of the circles associated with each word indicate that the generic category salmon (6), and the even more vague, but quite commonly cited, category of fish (6), both share the position of being the most cognitively salient prey "species" (in fact, species groups) to feature in lay people's mental representation of the ecosystem.

Bears appear especially important in people's web of beliefs, in that it is bears (with a betweenness centrality of 12) that emerge as primary conduits of caloric energy in this visual representation of people's responses. Salmon (7) and sea otters (5) also emerge as important in this regard, as do killer whales to a slightly lesser degree.

Note, however, that each of these features described above outlines people's implicit understanding of the system without accounting for the effects of time or effort, both of which actively constrain people's thinking in actual decision making contexts (Levine et al. 2015). To understand what comes to people's minds most easily, with the least amount of effort, in the least amount of time, note the relative size and density of the arrows. Namely, when we consider such cognitive accessibility, people's thinking about the system appears dominated by a specific handful of individual trophic relationships: bear $\rightarrow$ salmon, sea otter $\rightarrow$ urchin, wolf $\rightarrow$ deer, cougar $\rightarrow$ deer, sea otter $\rightarrow$ clam, killer whale $\rightarrow$ salmon, and killer whale $\rightarrow$ sea lion.

Together, this paints another picture, in which the general public's thinking about the local ecosystem revolves primarily around those trophic relationships that are most viscerally noticeable to the unaided human senses, i.e., without diving equipment, microscopes, or theoretical training in ecology. Thus, even if, for instance, wolves and cougars are not implicitly believed to be closely connected to the rest of the ecosystem, the eating habits of these charismatic megafauna nonetheless loom large in people's thinking. Crucially, because human attention and processing power is limited (Levine et al. 2015), this popular "habit of mind" (Atran 1998, Medin and Atran 2004) could be crowding out a conscious understanding of less obvious, yet structurally important, aspects of the regional food web.

\section{SPECULATION}

\section{Government managers}

To further explore the potential of our method, we also created a visualization based on interviews with four government managers (Fig. 2). The small sample size limits the figure's inferential power, but here we demonstrate how, even with a limited amount of data, this method can create an accessible image for use in hypothesis generation, or as a conversation starter in multistakeholder management contexts.

For instance, note that, although local residents identified a suite of predators as significant (Fig. 1), our government manager model suggests the sea otter (10), is by far the most central predator in managers' thinking. If borne out by further investigation, this could well signal a different locus of ecological attention among managers relative to the wider local population, a difference well worth considering in the course of any public consultation over the management of marine resources in the region.

Government managers also appear to display at least two key differences from the local civilian population with respect to their conceptions of prey. One, managers think at a higher phylogenic resolution than do locals. Note, for instance, the diversity of individual salmon species that emerge as salient in the managers' aggregate model, relative to local residents'. The second difference is that the most central prey species for managers is kelp (6), followed by urchin (3), and the category forage fish (3). This alerts us to the fact that government managers appear acutely cognizant of a distinct ecological subsystem that is entirely absent from locals' aggregate model: kelp beds.

Thus, by comparing the central prey species in managers' versus local residents' respective aggregate models, it quickly becomes clear that the two are characterized by a focus on largely different ecological subsystems and relationships. Although local civilians' aggregate mental model pivots largely around the consumption of fish species (particularly salmon) by a range of relatively easily observed, often charismatic megafauna, the managers' aggregate mental model is characterized by an awareness of several distinct ecological subsystems, none of which surfaces with the same degree of detail among locals' aggregate model. The human $\rightarrow$ salmonid $\Downarrow$ bear subsystem, the sea otter $\rightarrow$ invertebrate subsystem and vague awareness of the baleen whale $\rightarrow$ microscopic prey subsystem do feature in both managers' and locals' models. However, the former's model also includes a kelp-centered subsystem, a shark-centered subsystem, and a keen awareness of the role of two relatively unassuming predators that receive little to no attention in the locals' model (sunflower starfish and a shorebird known as an oystercatcher). The single species that links 
any of these subsystems together in the managers' model is the urchin, which receives the highest betweenness centrality score (1.583) of any species in their model.

This difference in cognitive accessibility of different features of the same shared ecosystem seems to us at least partly an artifact of the kinds of interactions and observational experiences that local residents, as opposed to managers, tend to have with their environment and vice versa. In the context of the often fraught relations between government resource managers and local residents in the Clayoquot Sound region, this apparent gulf in cognitive focus, specifically (as distinct from any simultaneous difference in environmental values), is a key point worthy of further testing, and consideration in management (Liu 2004, Van den Bossche et al. 2011).

\section{Males versus females}

Comparing the aggregate mental models of local resident men versus local resident women, respectively, also yields hypothesisgenerating insights. Note that the most central predators in male participants' aggregate model (Fig. 3) are, in descending order: bear (7); killer whale (7); and sea otter (6); followed by the relatively generic category salmon (3); as well as cougar (3); wolf (3); and halibut (3). For women, however, the most centrally prolific predator to emerge by far is human (12; see Fig. 4).

Although men did note, with a relatively low degree of saliency, that humans tend to eat "everything," it is the women's attention to the variety and specificity of human-ecosystem trophic relations that dominates the latter group's aggregate mental model. Although women did identify salmon as the most central prey species in the ecosystem (with an in-degree centrality score of 6), unlike men, they did not identify salmon, nor halibut, nor any other fish species, as important predators (see Fig. 4).

Although our data suggest there are certainly important similarities between men and women's mental models of the Clayoquot ecosystem, key differences in salience and content suggest that for men, salmon emerge as far more central, with a betweenness centrality score of 22.5 , whereas for women, it is sea otters (11), that take the relative, albeit considerably dimmer, spotlight. Although both genders appear cognizant of the same general array of relationships (e.g., bear $\rightarrow$ salmon, sea otter $\rightarrow$ invertebrates, human $\rightarrow$ many species, wolf and cougar $\rightarrow$ deer), there do appear to be consequential differences in the relative focus and resolution of men's ecological reasoning (i.e., fishcentered), versus women's (i.e., human- and otter-centered). Such evidence merits further investigation, and suggests the importance of acknowledging potential biases that could develop should either gender come to dominate consultative processes on marine resource management in the region (e.g., Johnson et al. 2004).

\section{First Nations versus non-First Nations}

Finally, dividing the local resident sample into First Nations and non-First Nations participants suggests even starker contrasts. In First Nations' aggregate mental model of the food web (Fig. 5), sea otter is by far the most central, prolific predator, represented as preying on eight different species of invertebrate, as well as on the general categories of shellfish and everything, for a total outdegree centrality score of 10 . Otters' perceived tendency to predate on clams, and urchins, in particular, appears especially salient for First Nations participants. In non-First Nations' aggregate
Fig. 3. Aggregate mental model of the regional food web: males. The feeding habits of large charismatic megafauna (bears, killer whales, cougars, and wolves) tend to dominate males' aggregate mental model in terms of cognitive accessibility. The prolific feeding habits of sea otters are also recognized but remain relatively less salient (with the slight exception of their consumption of urchins, and to a lesser extent crab). Salmon play the most central role in males' imagined trophic web: they are both the most important single prey species, as well as being most crucial to the transfer of energy throughout the food web. For men, other prey with particular importance to the wider food web include herring and clams. Humans are recognized as prolific predators, but with very low salience. (Note that this visualization is demonstrative of the method's potential uses, not necessarily representative of a demographic with statistically distinct responses).

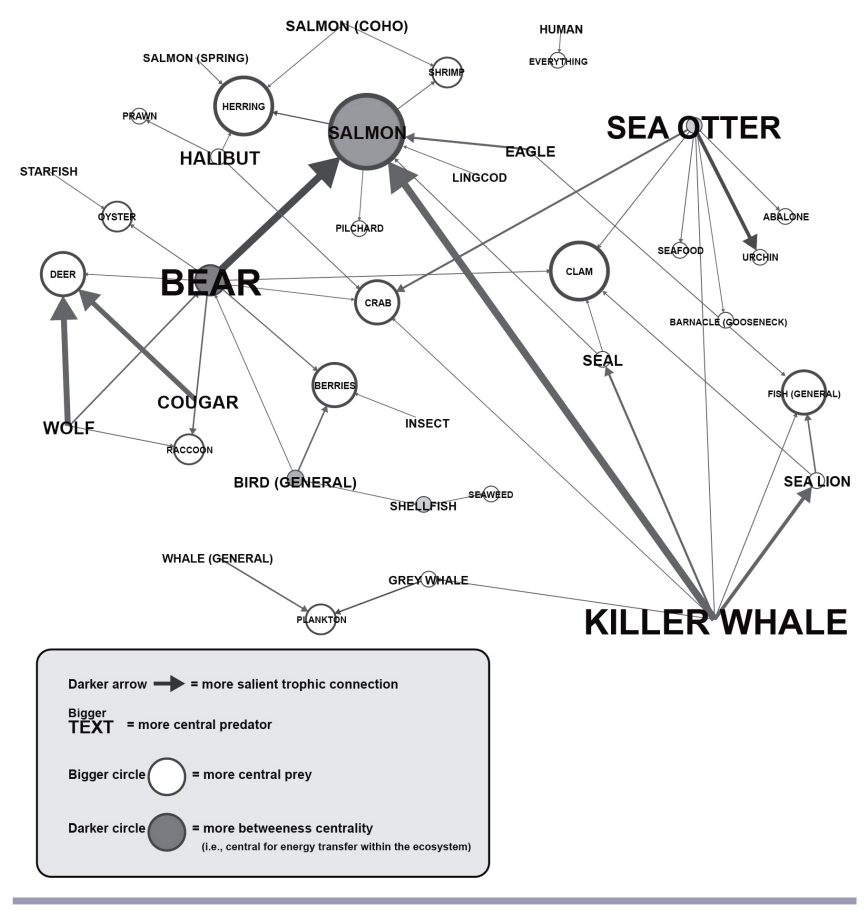

model, however (Fig. 6), humans are by far the more prolific, although not particularly salient, predator, boasting an outdegree centrality score of 12 . Sea otter, in contrast, receives a relatively low out-degree centrality score of five among non-First Nations, while otters' trophic connections also appear considerably less salient for non-First Nations than they do for First Nations.

Bear and killer whale appear as relatively central predators in both demographic groups' models, as does the general category salmon emerge as a shared central prey "species." However, on average, First Nations appear to find sockeye salmon, in particular, quite central (3), whereas non-First Nations appear less likely to think of salmonids at that high a resolution. For First Nations, clam, as a broad taxonomic group, is in fact the most central prey, with an in-degree centrality score of five. Non-First Nations, in 
Fig. 4. Aggregate mental model of the regional food web: females. Women's aggregate mental model is cognitively dominated by the feeding habits of sea otters. On average, women appear especially cognizant of otters' penchant for urchins and clams. Similarly salient for women are the feeding habits of large charismatic megafauna (bears, killer whales, cougars, and wolves). The feeding habits of humans appear to be less cognitively accessible to women than those of other mammals. Nonetheless, the prolific nature of human predation plays a uniquely central structural role in women's aggregate mental model. In other words, while humans' eating habits are not necessarily immediately apparent to women in the context of imagining the local food web, once humans' role is indeed considered, it becomes highly central to women's mental representation of the system. Central prey species in women's mental representations of the food web include salmon, oysters, and fish (the latter as a broad, but commonly cited, taxonomic category). Women also appear aware of several other parallel features of the food web, such as the importance of herring and krill to baleen whales. These subsystems nonetheless remain conceptually detached from the web as a whole. (Note that this visualization is demonstrative of the method's potential uses, not necessarily representative of a demographic with statistically distinct responses).

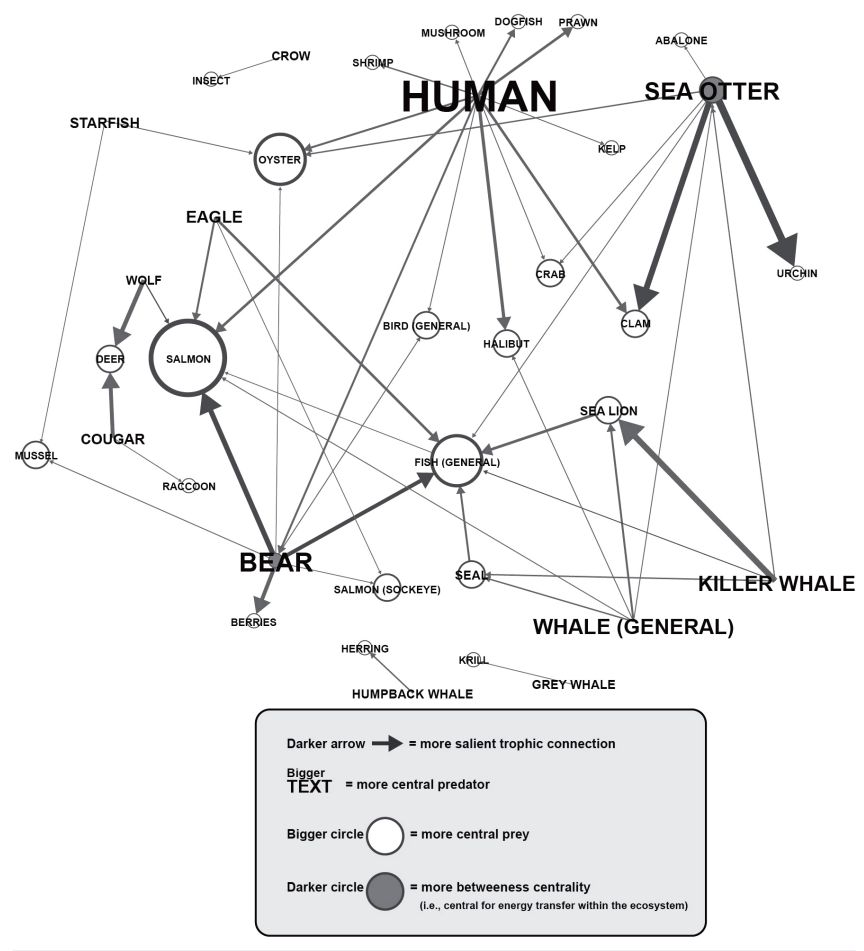

contrast, find the general category fish (6), as well as oyster (3) and plankton (3), to be more central as prey species than they do clam (2).

Remarkably, although sea otters are clearly the most central predator in First Nations' aggregate model, because they are apparently not readily thought of by First Nations as prey, it is instead salmon, seal, and sockeye salmon that feature as the most
Fig. 5. Aggregate mental model of the regional foodweb: First Nations. First Nations' aggregate model is cognitively dominated in terms of salience by the feeding habits of charismatic terrestrial predators (wolves, bears, cougars) as well as that of sea otters and, to a lesser extent, killer whales. Sea otters, in particular, emerge as especially central predators in First Nations' model, given the vast array of individual prey species otters are collectively believed to eat. Bears and killer whales are also regarded as relatively prolific predators, but it is their penchant for salmon that emerges as an especially important feature of First Nations' model. Salmon, in turn, are regarded by First Nations both as a central prey species, but also as marine predators in and of themselves. As such, for First Nations, salmon constitute the most central species in the food web with regard to facilitating energy transfer throughout the ecosystem. Seals occupy a similar, but slightly less central, role in First Nations' collective model. Humans are acknowledged as somewhat prolific predators, but with a low degree of salience. Baleen whales are noted for their predation on plankton and krill, but these relationships remain conceptually detached from the wider food web. Among First Nations, it appears that after salmon it is clams, specifically, that are regarded as the most widely targeted prey species in the ecosystem. (Note that this visualization is demonstrative of the method's potential uses, not necessarily representative of a demographic with statistically distinct responses).

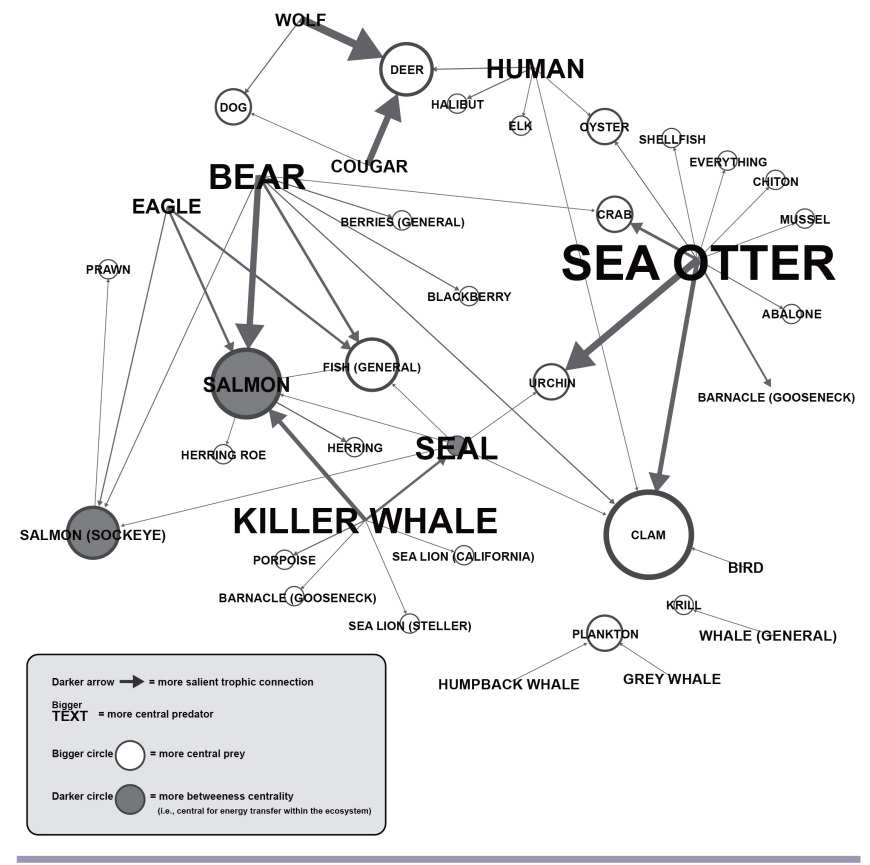

salient "connector" species for the food web as a whole, with betweenness centrality scores of 9.5, 5.5, and four, respectively. In non-First Nations' model, inversely, sea otter has a relatively high betweenness centrality (4), topped only by bear (8.5). Meanwhile salmon, although important, does not emerge as a particularly central connecting species in non-First Nations' model, partially because it is not readily regarded by non-First 
Fig. 6. Aggregate mental model of the regional food web: nonFirst Nations. For non-First Nations, the most salient aspects of the food web appear to be the predation habits of bears, killer whales, wolves, and cougars, as well as those of sea otters and sea lions. Humans feature as uniquely prolific predators, but not as particularly salient ones. Salmon, and fish in general, are regarded by non-First Nations as the most widely targeted, and hence central, prey species. However, non-First Nations seem relatively agnostic with respect to what those species in turn themselves eat. As such it is in fact bears, and birds, which emerge as the most central species to the foodweb in terms of energy transfer throughout the ecosystem. This is largely due to the fact that both are regarded as human prey, not true apex predators. Finally, non-First Nations appear somewhat uniquely cognizant of the feeding habits of baleen whales, with clear beliefs about how that whale-driven subsystem connects to the rest of the food web through herring and killer whale predation. (Note that this visualization is demonstrative of the method's potential uses, not necessarily representative of a demographic with statistically distinct responses).

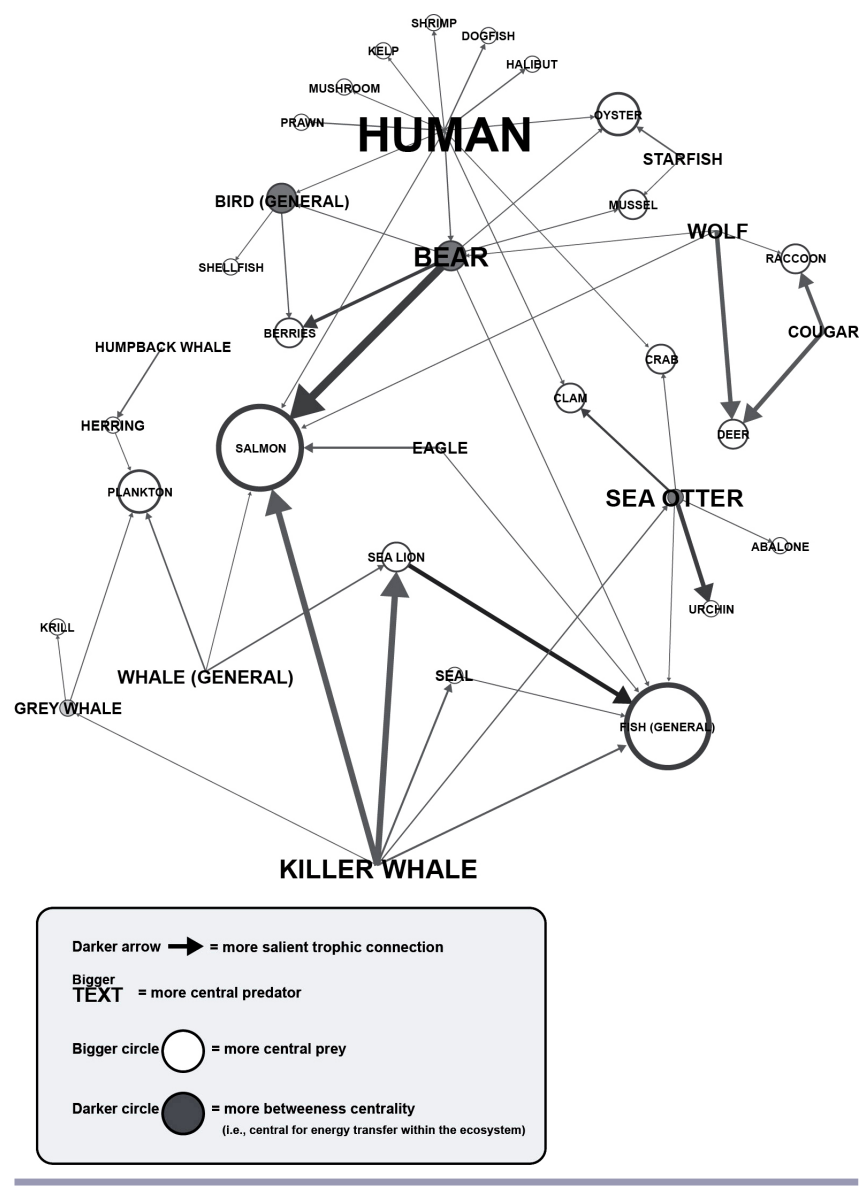

Nations as a predator. This latter point suggests that, on average, First Nations have much clearer, more salient beliefs about what salmon feed on than do non-First Nations.

In sum, although First Nations' and non-First Nations' models do suggest important areas of overlap, non-First Nations appear to think of humans, once they do think of them, as the central source of predation in the system. For First Nations, however, it is sea otters that loom cognitively as the most significant marine predator. And although both groups appear to think readily about salmon as a key prey species, and killer whales as an important predator, First Nations appear to think of the trophic interactions of both animals at a significantly higher phylogenetic resolution than do non-First Nations.

Together, these apparent differences hint at a number of possibilities. One, First Nations' comparative discounting of humans as predators, but relative stress on sea otters as predators, suggests there may be a subtle epistemic difference in terms of how First Nations and non-First Nations each tend, on average, to tacitly frame the human relationship to nature. Such effects have been reported in other cognitive anthropological studies contrasting the epistemic stance of indigenous and settler colonial peoples (Ross et al. 2007).

A second possibility is that differences between First Nations and non-First Nations participants' models of the local ecosystem may well constitute reflections of different habits of mind (Atran 1998, Medin and Atran 2004) acquired by virtue of differences in the two groups' economic and subsistence activities in the area. For instance, although our interview data suggest that, on average, non-First Nations spend their outdoors time in largely touristic, industrial, or recreational capacities, First Nations reported spending relatively more outdoors time devoted to subsistence food collection, which they are uniquely permitted to do by law.

This difference in habitual interaction with the ecosystem would help account both for the relatively higher phylogenetic resolution at which First Nations appear to think about key marine food species, e.g., sockeye salmon or nearshore edible invertebrates, as well as a higher degree of awareness of the various trophic interactions that support, or threaten, the particular food species themselves. From this perspective, it is also unsurprising that nonFirst Nations, conversely, seem more inclined to focus on the trophic relationships most readily apparent to the eye, and to the imagination, of a visitor, e.g., the feeding habits of charismatic species such as killer whales, bears, and wolves, without much need for attention to high resolution phylogenetic distinctions.

A third alternative is that both these above factors reinforce one another to create the sorts of observable differences among First Nations' and non-First Nations' aggregate mental models suggested by this speculative analysis. Given evidence for the pivotal role of shared mental models in negotiation processes (Liu 2004, Van den Bossche et al. 2011), we argue marine resource management negotiations among First Nations and non-First Nations actors could well stand to gain from actively investigating and considering the implications of such potential group-level differences.

\section{Potential uses of the method}

The above comparisons are based on demographic groups selected a prior. As such, they are speculative, overlap with one another, and thus do not necessarily constitute statistically robust axes of difference. However, in future applications, this limitation could be overcome by combining the method described here with one of several factor analyses. By employing, for instance, one or more forms of consensus analysis (e.g., Romney et al. 1987, Weller 
2007), researchers could explore whether given preidentified groups do in fact exhibit significantly different shared mental models (informal consensus analysis). Alternatively, the data could be rotated on various factors to determine if there are any tacit groups with significantly different response patterns present in a single sample. Both approaches could help reveal significant tacit drivers of conflict between groups, or, inversely, help debunk essentialist stereotypes that may be negatively affecting cooperation. Given this potential array of applications, as well as the hypothesis-generating capacity of the simpler approach demonstrated above, we suggest the outputs of this method could be well-suited for direct use in deliberative processes among stakeholders (e.g., Parkins and Mitchell 2005, Rodela 2012), or in participatory action research contexts (e.g., Johnson et al. 2004).

\section{CONCLUSION}

Mental models are receiving a growing amount of attention in the field of resource management (Jones et al. 2011, Lynam and Brown 2011, Lynam et al. 2012). However, their importance has yet to be fully integrated into multistakeholder management practice. This lack of full integration is relevant, because empirical research (Brodt and Dietz 1999, Liu 2004, Van den Bossche et al. 2011) suggests that it is actually the act of moving toward a convergent mental model itself that is most important in fostering good outcomes in negotiation contexts.

The method we detailed above helps facilitate this by combining salience analysis with network analysis. The result is an accessible but quantitatively robust representation of participants' collective mental model of a system, with an emphasis on relative cognitive salience. As suggested above, such outputs could have potential applications in both research and practice. There are however, some downsides to this method as it stands. One, although limited sample sizes can produce outputs that help generate hypotheses, small sample sizes can lead to visualizations that amplify the visceral impact of individual responses that are not necessarily reflective of shared cognitive terrain.

Two, because of time and cost limitations, we conducted only one iteration of the method with participants. Because people provided freelists of different lengths, this limited our ability make robust inferences about differences among individuals and subgroups. In future applications of this method, two iterations could be carried out to first generate a list of terms, and then use that same set of terms with each participant.

Three, without the integration of one or more forms of factor analysis into the method, it is not possible to statistically differentiate between groups of participants that have significant patterns of similarity and difference from one another. Time and financial constraints prevented us from doing so in the context of this first iteration of the method, but future applications would benefit from experimenting with various approaches to addressing this challenge.

Finally, we developed this method specifically for application to food webs, an important but limited domain. That said, with minor changes, the method could indeed be adapted to eliciting group-level mental models of any system that can be conceptualized as a network of nodes and edges. We thus encourage readers to consider adapting our method to a range of social-ecological contexts, iteratively modifying it to further develop the quality and inferential power of such mental-model visualizations.

Responses to this article can be read online at: http://www.ecologyandsociety.org/issues/responses. $\mathrm{php/8094}$

\section{Acknowledgments:}

We wish to thank NSERC, SSHRC, and the UBC Faculty of Graduate and Postdoctoral Studies for their support throughout the research and writing of this article.

\section{LITERATURE CITED}

Atran, S. 1998. Folk biology and the anthropology of science: cognitive universals and cultural particulars. Behavioural and Brain Sciences 21(4):547-569. http://dx.doi.org/10.1017/ s0140525x98001277

Barg, F. K., R. Huss-Ashmore, M. N. Wittink, G. F. Murray, H. R. Bogner, and J. J. Gallo. 2006. A mixed-method approach to understanding loneliness and depression in older adults. Journal of Gerontology B: Psychological Sciences 61(6):S329-S339. http:// dx.doi.org/10.1093/geronb/61.6.s329

Beratan, K. K. 2007. A cognition-based view of decision processes in complex social-ecological systems. Ecology and Society 12 (1):27. [online] URL: http://www.ecologyandsociety.org/vol12/ iss $1 / \operatorname{art} 271$

Borgatti, S. P. 1996. ANTHROPAC 4.0 [Computer Software]. Analytic Technologies, Natick, Massachusetts, USA.

Brandes, U. 2001. A faster algorithm for betweenness centrality. Journal of Mathematical Sociology 25(2):163-177. http://dx.doi. org/10.1080/0022250x.2001.9990249

Brodt, S. E., and L. E. Dietz. 1999. Shared information and information sharing: understanding negotiation as collective construal. Pages 263-283 in R. J. Bies, R. J. Lewicki, and B. H. Sheppard, editors. Research innegotiation in organizations Volume 7. Elsevier Science/JAI Press, Bingley, UK.

de Morais, F. F. 2009. Traditional ecological knowledge about plants cultivated by fishermen at Community Estirão Comprido, Pantanal matogrossense, Brazil. Boletim do Museu Paraense Emîlio Goeldi--Ciências humanas 4(2):277-294.

Dongre, A. 1R., and P. R. Deshmukh. 2012. Farmers' suicides in the Vidarbha region of Maharashtra, India: a qualitative exploration of their causes. Journal of Injury and Violence Research 4(1):2-6. http://dx.doi.org/10.5249/jivr.v4i1.68

Gephi. 2014. The Open Graph Viz Platform. The Gephi Consortium, Paris, France. [online] URL: https://gephi.org

Ghorbani, A., G. Langenberger, L. Feng, and J. Sauerborn. 2011. Ethnobotanical study of medicinal plants utilised by Hani ethnicity in Naban River Watershed National Nature Reserve, Yunnan, China. Journal of Ethnopharmacology 134(3):651-667. http://dx.doi.org/10.1016/j.jep.2011.01.011 
Gravlee, C. C., H. R. Bernard, C. R. Maxwell, and A. Jacobsohn. 2013. Mode effects in free-list elicitation: comparing oral, written, and web-based data collection. Social Science Computer Review 31(1):119-132. http://dx.doi.org/10.1177/0894439312455312

Green, D. W., S. J. Muncer, T. Heffernan, and I. C. McManus. 2003. Eliciting and representing the causal understanding of a social concept: a methodological and statistical comparison of two methods. Papers on Social Representations 12:2.1-2.23.

Janssen, M. A., Ö. Bodin, J. M. Anderies, T. Elmqvist, H. Ernstson, R. R. J. McAllister, P.Olsson, and P. Ryan. 2006. Toward a network perspective on the resilience of social-ecological systems. Ecology and Society 11(1):15. [online] URL: http://www. ecologyandsociety.org/vol11/iss1/art15/

Johnson, N., N. Lilja, J. A. Ashby, and J. A. Garcia. 2004. The practice of participatory research and gender analysis in natural resource management. Natural Resources Forum 28(3):189-200. http://dx.doi.org/10.1111/j.1477-8947.2004.00088.x

Jones, N. A., H. Ross, T. Lynam, P. Perez, and A. Leitch. 2011. Mental models: an interdisciplinary synthesis of theory and methods. Ecology and Society 16(1):46. [online] URL: http:// www.ecologyandsociety.org/vol16/iss1/art46/

Levine, J., K. M. A. Chan, and T. Satterfield. 2015. From rational actor to efficient complexity manager: exorcising the ghost of Homo economicus with a unified synthesis of cognition research. Ecological Economics 114:22-32. http://dx.doi.org/10.1016/j. ecolecon.2015.03.010

Liu, L. A. 2004. Shared mental models in negotiation. Graduate School of Vanderbilt University, Nashville, Tennessee, USA.

Lynam, T., and K. Brown. 2011. Mental models in humanenvironment interactions: theory, policy implications, and methodological explorations. Ecology and Society 17(3):24. http://dx.doi.org/10.5751/ES-04257-170324

Lynam, T., R. Mathevet, M. Etienne, S. Stone-Jovicich, A. Leitch, N. Jones, H. Ross, D. Du Toit, S. Pollard, H. Biggs, and P. Perez. 2012. Waypoints on a journey of discovery: mental models in human-environment interactions. Ecology and Society 17(3):23 http://dx.doi.org/10.5751/es-05118-170323

Malan, D. F., and D. F. R. Neuba. 2011. Traditional practices and medicinal plants use during pregnancy by Anyi-Ndenye Women. African Journal of Reproductive Health 15(1):85-93.

Medin, D. L., and S. Atran. 2004. The native mind: biological categorization and reasoning in development and across cultures. Psychological Review 111(4):960-983. http://dx.doi. org/10.1037/0033-295x.111.4.960

Parkins, J. R., and R. E. Mitchell. 2005. Public participation as public debate: a deliberative turn in natural resource management. Society and Natural Resources 18(6):529-540. http://dx.doi.org/10.1080/08941920590947977

Pradhan, M. R., and U. Ram. 2010. Perceived gender role that shape youth sexual behaviour: evidence from rural Orissa, India. Journal of Adolescence 33(4):543-551. http://dx.doi.org/10.1016/ j.adolescence.2009.10.014
Rodela, R. 2012. Advancing the deliberative turn in natural resource management: an analysis of discourses on the use of local resources. Journal of Environmental Management 96 (1):26-34. http://dx.doi.org/10.1016/j.jenvman.2011.10.013

Romney, A. K., W. H. Batchelder, and S. C. Weller. 1987. Recent applications of cultural consensus theory. American Behavioral Scientist 31(2):163-177. http://dx.doi.org/10.1177/000276487031002003

Ross, N., D. Medin, and D. Cox. 2007. Epistemological models and culture conflict: Menominee and Euro-American hunters in Wisconsin. Ethos 35(4):478-515. http://dx.doi.org/10.1525/ eth.2007.35.4.478

Smith, J. J., and S. P. Borgatti. 1997. Salience counts-and so does accuracy: correcting and updating a measure for free-list-item salience. Journal of Linguistic Anthropology 7(2):208-209. http:// dx.doi.org/10.1525/jlin.1997.7.2.208

Sutrop, U. 2001. List task and a cognitive salience index. Field Methods 13(3):263-276. http://dx.doi.org/10.1177/1525822x0101300303

Thompson, E. C., and Z. Juan. 2006. Comparative cultural salience: measures using free-list data. Field Methods 18 (4):398-412. http://dx.doi.org/10.1177/1525822x06293128

Van den Bossche, P., W. Gijselaers, M. Segers, G. Woltjer, and P. Kirschner. 2011. Team learning: building shared mental models. Instructional Science 39(3):283-301. http://dx.doi.org/10.1007/ $\underline{\text { s11251-010-9128-3 }}$

Weller, S. C. 2007. Cultural consensus theory: applications and frequently asked questions. Field Methods 19(4):339-368. http:// dx.doi.org/10.1177/1525822X07303502 


\section{Appendix 1}

\section{Note on mental models versus cultural models:}

Group-level models are sometimes referred to as 'cultural models' (Kronenfeld 2008). However, for the purposes of this paper, we prefer the term 'aggregate' mental model, in that we mean our term to refer more specifically to a collection of individual mental models that may or may not feature a high degree of agreement amongst them, but nonetheless collectively represent the cumulative system of beliefs of a given group of individuals that have been identified or selected a priori to constitute a single unit. Such groups may emerge by institutional arrangement (e.g., political circumstance creating 'strange bedfellows' or alliances of dissimilar individuals that nonetheless go on to work together as a single actor, guided by their cumulative set of beliefs), by research logic (as in the case of this paper), or otherwise. 


\section{Appendix 2}

Figure A2.1: Map of Clayoquot Sound, British Columbia, Canada

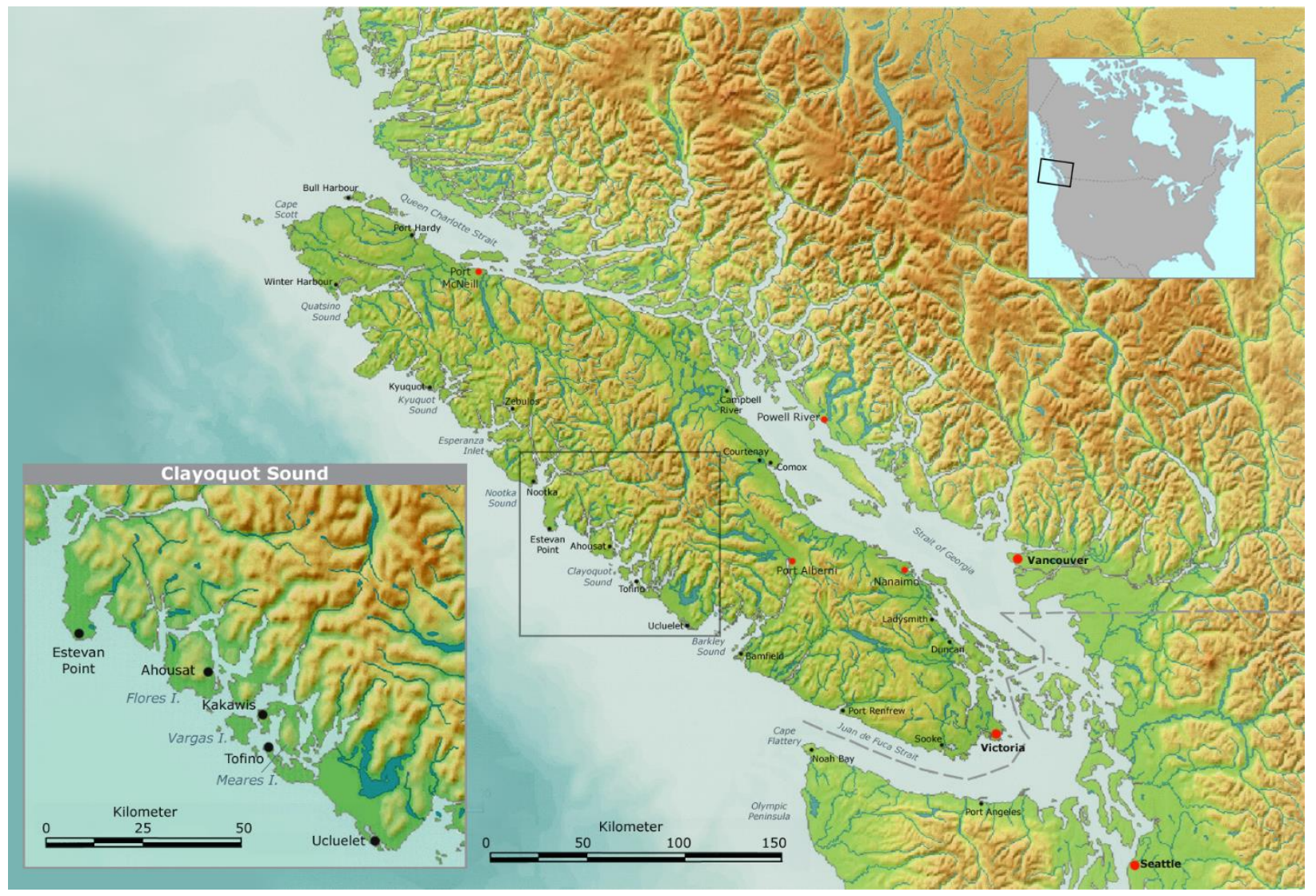

(Public domain, via Wikimedia) 


\section{Appendix 3}

\section{Full interview protocol}

\section{Part 1 -- Mental Model Basics:}

\section{Associative Networks and Analogical Representations of WCVI region and ecology}

I - Identity, location and spatial cognition

1) “May I ask .... your name?” ... [Optional “...where and when were you born?”]

2) "Where do you live and/or work (and/or are you visiting)?"

3) "What do you do / are you doing (t)here?" ... "What's involved in your line of work?" ... "How long have you been doing [main activity $X$ ]?" ... "What part(s) of the coast does [main activity $X$ ] take you to?" ... "Are there any other kinds of activities you engage in regularly on the west coast?" ... "Do you use or harvest the resources in any other way?"

Additional projection tasks:

4) "Would you like to show me, using these drawing utensils and notepad, the area where we are now / where you live/work / where you('ve) come to visit?" [Prompt for explanations] Photograph.

5) [Show map of west coast / specific sub-region] - "Please tell me what you see here. Are there any particularly important areas for you? Particularly important places? What are they?" [Make a list, and/or mark on the map] ... "Why are they important?" [Extra time: prompt for stories] - Photograph.

\section{II -- Most accessible associations with place}

1) "What do you like about living / working / visiting here (on the west coast of the island)?"

2) "What do you dislike about living / working / visiting here (on the west coast of the island)?"

3) "What are the 'problems' or 'challenges' in this area that you drew / described? What is [problem or challenge $x$ or $y$ ] 'like'? How would you describe it to someone who knew nothing about [issue $\mathrm{x}$ or $\mathrm{y}]$ ?"

Prompt for specifics. 


\section{III - Most accessible species/resources; associations and mental models of their interrelationships; additional focus on trophic cascade species}

1) "Now let's make a list of species (plants and animals) / resources on the west coast of Vancouver Island that come to mind / that are important to you."

[have the participant write down the name of each species on an individual flashcard or piece of paper; record the order in which these species are listed]

1a) [Optional] "How would you arrange these species/flashcards ... into groups ... or in a line?" [Can ask for both kinds of arrangement, one at a time] "Please do so." [Prompts and optional forced orderings: "Which species 'go together by nature'?" (via Atran et al.) ... What's related to what ... Most tasty to least tasty .... Most abundant to least abundant ... Most valuable to least valuable ... Most expensive to least expensive... Most important to least important ... What lives where ... ask for explanations as they do these sorts] - Photograph for each arrangement.

2) Present popsicle sticks, with 'eats $\rightarrow$ ' ... "Now let's build a food web... please show me/tell me what eats what," ... ask for descriptions as participant builds food web. If possible, in inoffensive way, ask how they know, or why they guess one way or another, how sure they are, about each trophic interaction ... - Photograph.

3) Extra focus on associations/beliefs pertaining to sea otters, if they have not already come up: "What can you tell me about sea otters?" ... "What do sea otters eat?" ... "What effect do sea otters have on the ecosystem / other species?" ... "How do you feel about sea otters?" ... "Do you have any stories about sea otters?" ... "Do you think sea otters should be managed?" ... "Can sea otters be managed?"” ... etc.

4) Extra focus on associations/beliefs pertaining to kelp forests, if they have not already come up: "What can you tell me about kelp (forests)?" ... "What role do kelp forests play in the ecosystem?" ... "What effect do they have on other species" ... "How do you feel about kelp forests?" ... "Do you have any stories about kelp (forests)?" ... etc.

If the participant has not done so themselves, you can ask participants if they distinguish between different kinds of kelp, and ask similar questions about the different kinds.

5) "Now let's talk about health and well-being, of the ecosystem, and/or of these species." [Point to flashcards already laid out] ... "What sort of things tell you if the ecosystem, or the species/resources that are important for you, are (un)healthy?" ... "What are your 'indicators'?" [Prompt: E.g. When there's an algae bloom, what does that mean about the ecosystem? ... or ... How do you know if its going to be a good year/season for species $x$ or $y$ ?] [Optional: listand/or add flashcards for these--Photograph] ... Why are these your indicators? How does this tell you about the ecosystem? I.e. How does indicator $\mathbf{x}$ or $\mathbf{y}$ affect species $\mathbf{z}$, or vice versa? [This prompts for theories and mental models of the ecosystem dynamics in addition to the food web]

6) "What else affects the health of these species/resources?" ... "Are there any natural (or other) processes that affect these species/resources/the ecosystem?" ... [Prompt: E.g. change in seasons, El Nino, migrations, things that occur in remote regions such as fishing pressure or ecological conditions in Alaska, human activities etc.] Prompt for underlying mental models. 
IV - Most accessible changes; associations and mental models relating to these processes; historical cognition

[Depending on time, do this either before, or after, the following section, according to priority]

1) "Now let's talk about changes. What are some of the biggest changes you see happening on the west coast, in the past, now, or in the future?" ... I.e. important changes that have happened, are happening, or probably will happen. [Can ask for each of these separately] [Prompt: ecological changes, policy changes, social/socioeconomic changes, ... More specifically: Sea otter reintroduction, climate change, development, deforestation, salmon or otherfishery depletion, recent salmon returns, changes in fishery legislation, invasive species, newspecies sightings, etc.] ... [Optional: add flashcards in for these changes] ... If possible, inoffensively ask how they know: "What is it that tells you these changes are happening / have happened?" [If they cite ecological indicators, query about the underlying mental model]

2) "How do you feel about (each of) these changes?" ... "How will they affect the ecosystem, or what's important to you in that system?" ... "What are your hopes or fears about what will happen to the ecosystem and/or your community into the future?"

3) Optional: "Going back as far as you like, what have been the most significant events, in your mind, here on the WCVI?" ... Prompt: e.g. arrival of Europeans, collapse of salmon fishery, etc.

\section{Part 2 -- Focusing on the human element:}

\section{Associative networks and analogical representations of user groups / stakeholders and their interrelationships with the WCVISES}

V - Most accessible user groups/stakeholders; associations and mental models

1) "Now let's talk a bit more about people, specifically." ... "How do people affect this foodweb/ecosystem?" ... [point to earlier flashcard arrangement of species/resources/habitats/foodweb] ..."Please describe."

2) "Who are the important resource users / stakeholders / individuals / groups of people /organizations that affect this ecosystem and vice versa?" ... "Let's make a list." [Optional: add in flashcards for these names]-Photograph.

2a) Optional: [For each 'stakeholder' flashcard] - "What (thee-to-five) words would you use to describe/do you associate with this flashcard [i.e. stakeholder]? What makes them important?" [Extra time: "Anything else you want to add? Any stories about these stakeholders/people?"]

3) "How do you think these different stakeholders/groups see the ecosystem(s) of the west coast?" ... "Do they each see things the way you do?" ... "What might be the differences?" ... 
"Do they know what you know, or vice versa?" ... "Why or why not?" ... "Should they?" [Prompt for theories and beliefs about these other stakeholders]

4) Optional: "How would you arrange these 'people' flashcards ... into groups ... or in a line?" [Can ask for both, one at a time] ... "Please do so." [Prompts and optional forced orderings: Which affect your life/business the most, versus the least? ... Most trustworthy to least trustworthy ... Biggest effect on the ecosystem to smallest effect ... Most transparent to least transparent ... Most familiar to you to least familiar to you ... Most powerful to least powerful] Photograph (for each arrangement).

\section{Part 3 - Ecosystem Services}

$\mathrm{VI}$ - Most accessible ecosystem services and their function in mental models

1) "Now we're going to talk a bit more about the ecosystem/nature, and its relationship to people." ... "What things does the ecosystem/nature do for people? What does it provide for people?" ... "Let's make a list" ... [Optional: add flashcards for these] - Photograph.

1a) Optional: "How would you order these things?" ... E.g. from most important to least important - Photograph.

2) "You mentioned changes $\mathbf{x}, \mathbf{y}, \mathbf{z}$ above ... do you think those changes might affect any of these things (i.e. the list of ecosystem services) in the future?" ... "How?"

\section{Part 4 - Policies and parks status}

\section{VI - Most accessible government policies; associations and role in mental models}

1) "Now let's talk briefly about the role of government and regulation here on the west coast" ... "Are there any government policies or actions (past or present) that come to mind (federal, provincial, local) as important to you or your life here on the west coast?" ... If so: "Let's make a list." [Optional: add flashcards] 
2) For each policy: "What can you tell me about this policy?" ... "How does it work?" ... "How does it affect you?" ... Prompt for explanations of the policy mechanisms: e.g. how the fishing license buy-back worked, why it was put in, etc. ... "How do you feel about it?" ... "Why do you think this policy was put in place?" ... "Is it a good thing? A bad thing? Why?"

3) Optional: "Did you know the Clayoquot Sound region is a 'Biosphere Reserve'?" ... "What does that mean to you?" ... "What is a Biosphere Reserve? How would you describe it to someone who knows nothing about Biosphere Reserves or the west coast?" ... "Has it had any major successes/accomplishments?" ... “Do you consider it a success?" ... "Why/why not?"

4) Optional: "What do you think about the protected areas on the west coast?" ... E.g. the Pacific Rim Park Reserve? [Others?] ... "Are they important to you / do they affect you?" ... "If so, why?"

\section{Part 5 - Information sources [optional - discretionary]}

\section{VII - Social cognition}

"Now, if you're willing, I have a few more personal-sounding questions that pertain to who you are as a participant, and how knowledge gets passed on... you're free, of course, to answer these, or not, as you feel comfortable"

1) "Where did you learn about [main activity $X$, e.g. fishing]?" ... "How did you learn?" ... "Who taught you?"

2) "If or when you have questions about certain species or resources / the ecosystem / certain policies, where do you turn / who do you turn to for information, advice or clarification?"

3) “Do you have, or have you had, 'role models,' or people you 'look up to' or 'turn to,' in life, or with respect to [main activity $X$ ], or life in general?" ... alternatively: "Who would you say has had the biggest influence on your [working] life / how you view life?" ... "Why?"

4) "Do you have any particular places you like to go to unwind / socialize with other people?" ... "Where are they, if you don't mind my asking?" ... Can ask about what kinds of conversation go on in these places, who / which kinds of people tend to be there.

\section{VIII - Cultural cognition}

1) "Where do you get information about what's happening in your community, or on the west coast?"

2) Optional: "Do you listen to the radio / watch T.V.?" ... “Do you have any favourite channels or programs?" ... "Do you read much?" ... "If so, what sorts of things do you like to read?" ... "Have 
you ever read anything, or seen any movies, that have really shaped the way you see things?" ... "If so, what / how?"

\section{Part 6 - Specific associations [optional]}

1) "What [kinds of things] do these terms mean to you [if at all]?" ... "What [one, three or five] word(s) do you associate with them / would you use to describe them?" Prompt: e.g. if I say 'banana', you say..?

[Development; Conservation; Sustainability; Sustainable development; Biosphere reserve; Ecosystem services; Science; Traditional knowledge; Sea otters (if this hasn't come up already); Kelp (same as above); DFO (same as above)]

\section{END}

(If there is extra time, go back over questions and ask those marked 'optional' or 'extra time' that weren't already asked)

Thank participant for their time. Remind them about contact information in case they would like to see any outputs of the study. 


\section{Appendix 4}

\section{Species salience data derived from the first freelisting task of the protocol}

This is based on the formula:

$S=\left(\left(\Sigma\left(L-R_{\mathrm{j}}+1\right)\right) / L\right) / N$

where $S$ is the average rank of an item across all lists in the sample, weighted by the lengths of the lists in which the item actually occurs; $L=$ the length of (number of items in) a list; $R_{\mathrm{j}}=$ the rank of an item in the list (first = 1); and $N=$ the number of lists in the sample" (Smith and Borgatti 1997, 208-209).

(Table lengths reflect the point at which notable patterns of differences are observable in the results)

\begin{tabular}{|l|c|c|}
\hline SPECIES & $\begin{array}{c}\text { RANK } \\
\text { BY S }\end{array}$ & $\begin{array}{c}\text { SMITH'S SALIENCE } \\
\text { INDEX (S) }\end{array}$ \\
\hline Bear & 1 & 0.480 \\
\hline Halibut & 2 & 0.444 \\
\hline Cougar & 3 & 0.401 \\
\hline Wolf & 4 & 0.394 \\
\hline Eagle & 5 & 0.377 \\
\hline Sea otter & 6 & 0.367 \\
\hline Salmon & 7 & 0.366 \\
\hline Clam & 8 & 0.309 \\
\hline Sea lion & 9 & 0.291 \\
\hline Grey whale & 10 & 0.285 \\
\hline
\end{tabular}

\begin{tabular}{|l|c|c|}
\hline SPECIES & $\begin{array}{c}\text { RANK } \\
\text { BY S }\end{array}$ & $\begin{array}{c}\text { SMITH'S SALIENCE } \\
\text { INDEX }(S)\end{array}$ \\
\hline Giant kelp & 1 & 0.712 \\
\hline Sea otter & 2 & 0.624 \\
\hline Bull kelp & 3 & 0.619 \\
\hline Chinook salmon & 4 & 0.480 \\
\hline Sockeye salmon & 5 & 0.459 \\
\hline Coho salmon & 6 & 0.448 \\
\hline Chum salmon & 7 & 0.432 \\
\hline Sea lettuce & 8 & 0.411 \\
\hline Abalone & 9 & 0.406 \\
\hline Humpback whale & 10 & 0.404 \\
\hline
\end{tabular}

Table A4.1 Most cognitively accessible species: civilian locals (top 10)

Table A4.2 Most cognitively accessible species: government managers (top 10) 


\begin{tabular}{|l|c|c|}
\hline SPECIES & $\begin{array}{c}\text { RANK BY } \\
\text { S }\end{array}$ & $\begin{array}{c}\text { SMITH'S } \\
\text { SALIENCE } \\
\text { INDEX (S) }\end{array}$ \\
\hline Bear & 1 & 0.587 \\
\hline Cougar & 2 & 0.575 \\
\hline Wolf & 3 & 0.572 \\
\hline Eagle & 4 & 0.449 \\
\hline Sea otter & 5 & 0.422 \\
\hline Grey whale & 6 & 0.371 \\
\hline Urchin & 7 & 0.367 \\
\hline Clam & 8 & 0.364 \\
\hline Oyster & 8 & 0.364 \\
\hline Sea lion & 9 & 0.345 \\
\hline Crow & 10 & 0.345 \\
\hline Gull & 11 & 0.323 \\
\hline Killer whale & 12 & 0.302 \\
\hline Crab & 13 & 0.301 \\
\hline Halibut & 14 & 0.294 \\
\hline Steller's jay & 15 & 0.293 \\
\hline Salmon & 16 & 0.284 \\
\hline Humpback whale & 16 & 0.284 \\
\hline Whale & 17 & 0.272 \\
\hline Deer & 18 & 0.254 \\
\hline
\end{tabular}

Table A4.3 Most cognitively accessible species: females (top 20)

\begin{tabular}{|l|c|c|}
\hline SPECIES & $\begin{array}{c}\text { RANK } \\
\text { BY S }\end{array}$ & $\begin{array}{c}\text { SMITH'S } \\
\text { SALIENCE } \\
\text { INDEX (S) }\end{array}$ \\
\hline Halibut & 1 & 0.558 \\
\hline Salmon & 2 & 0.429 \\
\hline Bear & 3 & 0.398 \\
\hline Lingcod & 4 & 0.369 \\
\hline Sea otter & 5 & 0.325 \\
\hline Eagle & 6 & 0.322 \\
\hline Coho salmon & 7 & 0.289 \\
\hline Sockeye salmon & 8 & 0.283 \\
\hline Clam & 9 & 0.267 \\
\hline Cougar & 9 & 0.267 \\
\hline Wolf & 10 & 0.258 \\
\hline Killer whale & 11 & 0.255 \\
\hline Sea lion & 12 & 0.250 \\
\hline Deer & 13 & 0.242 \\
\hline Rockfish & 14 & 0.239 \\
\hline Crab & 15 & 0.237 \\
\hline Herring & 16 & 0.225 \\
\hline Grey whale & 17 & 0.219 \\
\hline Yelloweye rockfish & 17 & 0.219 \\
\hline Urchin & 18 & 0.212 \\
\hline
\end{tabular}

Table A4.4 Most cognitively accessible species: males (top 20) 


\begin{tabular}{|l|c|c|}
\hline SPECIES & $\begin{array}{c}\text { RANK } \\
\text { BY S }\end{array}$ & $\begin{array}{c}\text { SMITH'S } \\
\text { SALIENCE } \\
\text { INDEX (S) }\end{array}$ \\
\hline Halibut & 1 & 0.467 \\
\hline Bear & 2 & 0.432 \\
\hline Wolf & 3 & 0.418 \\
\hline Urchin & 4 & 0.406 \\
\hline Cougar & 5 & 0.405 \\
\hline Sockeye salmon & 6 & 0.397 \\
\hline Deer & 7 & 0.395 \\
\hline Clam & 8 & 0.361 \\
\hline Eagle & 9 & 0.333 \\
\hline Sea otter & 10 & 0.264 \\
\hline Killer whale & 11 & 0.241 \\
\hline Oyster & 12 & 0.237 \\
\hline Salmon & 13 & 0.231 \\
\hline Chiton & 14 & 0.229 \\
\hline Coho salmon & 15 & 0.226 \\
\hline Crab & 16 & 0.224 \\
\hline Spring salmon & 17 & 0.221 \\
\hline Crow & 18 & 0.206 \\
\hline Herring & 19 & 0.186 \\
\hline Cod & 19 & 0.186 \\
\hline
\end{tabular}

\begin{tabular}{|l|c|c|}
\hline SPECIES & $\begin{array}{c}\text { RANK } \\
\text { BY S }\end{array}$ & $\begin{array}{c}\text { SMITH'S } \\
\text { SALIENCE } \\
\text { INDEX (S) }\end{array}$ \\
\hline Bear & 1 & 0.510 \\
\hline Salmon & 2 & 0.452 \\
\hline Sea otter & 3 & 0.432 \\
\hline Halibut & 4 & 0.429 \\
\hline Eagle & 5 & 0.405 \\
\hline Cougar & 6 & 0.398 \\
\hline Wolf & 7 & 0.379 \\
\hline Sea lion & 8 & 0.375 \\
\hline Grey whale & 9 & 0.357 \\
\hline Gull & 10 & 0.324 \\
\hline Oyster & 11 & 0.297 \\
\hline Killer whale & 11 & 0.297 \\
\hline Crab & 12 & 0.290 \\
\hline Humpback whale & 13 & 0.281 \\
\hline Clam & 14 & 0.276 \\
\hline Lingcod & 15 & 0.269 \\
\hline Crow & 16 & 0.265 \\
\hline Whale & 17 & 0.254 \\
\hline Red cedar & 18 & 0.230 \\
\hline Hemlock & 20 & 0.229 \\
\hline
\end{tabular}

Table A4.5 Most cognitively accessible species: First Nations

(top 20)

Table A4.6 Most cognitively accessible species: non-First Nations (top 20) 


\section{Appendix 5}

Figure A5.1: Sample output of a completed trophic connection task

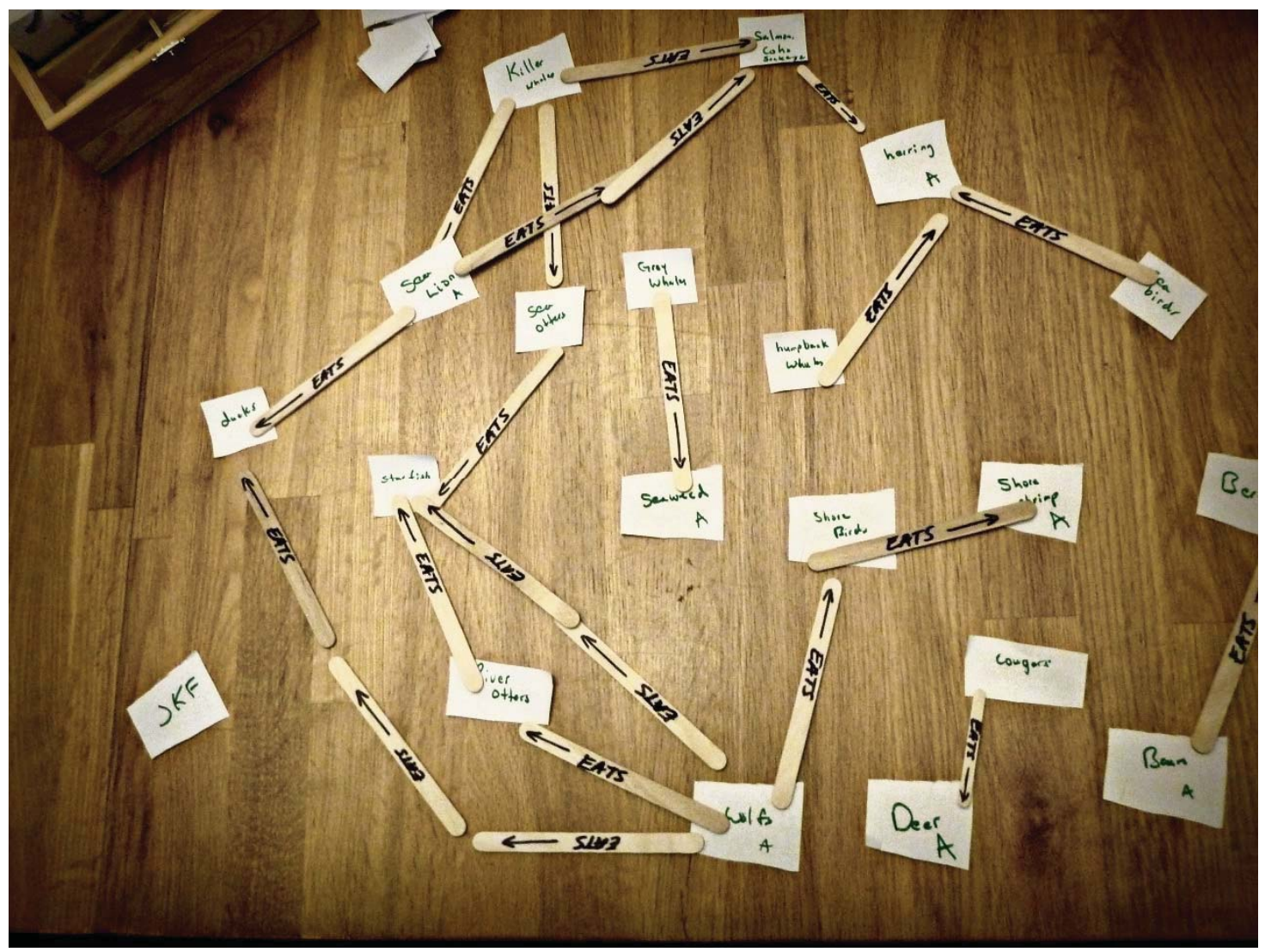

This image is one example of the final output of the freelisting and trophic connection tasks put to participants in the course of the interview protocol. Participants were first asked to list as many local species as possible, as they were able to think of them. The order of each participant's listing of species was recorded for later salience analysis. Participants were then prompted to depict what they believed to be those listed species' trophic interactions with other species, using a large number of popsicle sticks with the word "Eats $\rightarrow$ " that we subsequently provided to them. The order in which each participant connected each species one to another was also then recorded for later salience analysis. Finally, an image was taken of the result. 


\section{Appendix 6}

Trophic-relationship salience data derived from the second freelisting task of the protocol

(Table lengths reflect different points at which notable patterns of differences are observable in the results)

\begin{tabular}{|l|c|c|}
\hline $\begin{array}{l}\text { PREDATOR } \rightarrow \text { PREY } \\
\text { RELATIONSHIP }\end{array}$ & $\begin{array}{c}\text { RANK } \\
\text { BY S }\end{array}$ & $\begin{array}{c}\text { SMITH'S } \\
\text { SALIENCE } \\
\text { INDEX (S) }\end{array}$ \\
\hline Bear $\rightarrow$ Salmon & 1 & 0.230 \\
\hline Wolf $\rightarrow$ Deer & 2 & 0.219 \\
\hline Sea otter $\rightarrow$ Urchin & 3 & 0.191 \\
\hline Cougar $\rightarrow$ Deer & 4 & 0.185 \\
\hline Killer whale $\rightarrow$ Salmon & 5 & 0.175 \\
\hline Killer whale $\rightarrow$ Sea lion & 6 & 0.147 \\
\hline Sea otter $\rightarrow$ Clam & 7 & 0.133 \\
\hline Sea lion $\rightarrow$ Fish & 8 & 0.109 \\
\hline Eagle $\rightarrow$ Salmon & 8 & 0.109 \\
\hline Bear $\rightarrow$ Berries & 9 & 0.107 \\
\hline Killer whale $\rightarrow$ Seal & 10 & 0.103 \\
\hline Sea otter $\rightarrow$ Crab & 11 & 0.090 \\
\hline Bear $\rightarrow$ Fish & 12 & 0.088 \\
\hline Eagle $\rightarrow$ Fish & 13 & 0.086 \\
\hline Cougar $\rightarrow$ Raccoon & 14 & 0.082 \\
\hline
\end{tabular}

\begin{tabular}{|l|c|c|}
\hline $\begin{array}{l}\text { PREDATOR } \rightarrow \text { PREY } \\
\text { RELATIONSHIP }\end{array}$ & $\begin{array}{c}\text { RANK } \\
\text { BY S }\end{array}$ & $\begin{array}{c}\text { SMITH'S } \\
\text { SALIENCE } \\
\text { INDEX (S) }\end{array}$ \\
\hline Human $\rightarrow$ Chinook salmon & 1 & 0.250 \\
\hline Sea otter $\rightarrow$ crab & 1 & 0.250 \\
\hline Sea otter $\rightarrow$ Sea cucumber & 1 & 0.250 \\
\hline Bear $\rightarrow$ Berries & 1 & 0.250 \\
\hline Sea otter $\rightarrow$ Mussel & 2 & 0.242 \\
\hline Bear $\rightarrow$ Leaves & 2 & 0.242 \\
\hline Human $\rightarrow$ Chum salmon & 3 & 0.240 \\
\hline Sea otter $\rightarrow$ Swm. Scallop & 4 & 0.239 \\
\hline Abalone $\rightarrow$ Kelp & 5 & 0.234 \\
\hline Sea otter $\rightarrow$ Gnk. Barnacle & 5 & 0.234 \\
\hline Human $\rightarrow$ Sockeye salmon & 6 & 0.231 \\
\hline Sea otter $\rightarrow$ Butter clam & 7 & 0.227 \\
\hline Red urchin $\rightarrow$ Kelp & 7 & 0.227 \\
\hline Sea otter $\rightarrow$ Chiton & 7 & 0.227 \\
\hline Human $\rightarrow$ Coho salmon & 8 & 0.221 \\
\hline
\end{tabular}

Table A6.1 Most cognitively accessible trophic relationships: civilian locals

(top 15)

Table A6.2 Most cognitively accessible trophic relationships: government managers (top 15) 


\begin{tabular}{|l|c|c|}
\hline $\begin{array}{l}\text { PREDATOR } \rightarrow \text { PREY } \\
\text { RELATIONSHIP }\end{array}$ & $\begin{array}{c}\text { RANK } \\
\text { BY S }\end{array}$ & $\begin{array}{c}\text { SMITH'S } \\
\text { SALIENCE } \\
\text { INDEX (S) }\end{array}$ \\
\hline Sea otter $\rightarrow$ Urchin & 1 & 0.264 \\
\hline Sea otter $\rightarrow$ Clam & 2 & 0.224 \\
\hline Wolf $\rightarrow$ Deer & 3 & 0.198 \\
\hline Bear $\rightarrow$ Salmon & 4 & 0.184 \\
\hline Cougar $\rightarrow$ Deer & 5 & 0.180 \\
\hline Bear $\rightarrow$ Berries & 6 & 0.163 \\
\hline Killer whale $\rightarrow$ S. lion & 7 & 0.159 \\
\hline Bear $\rightarrow$ Fish & 7 & 0.159 \\
\hline Human $\rightarrow$ Halibut & 8 & 0.145 \\
\hline Sea lion $\rightarrow$ Fish & 9 & 0.132 \\
\hline Human $\rightarrow$ Salmon & 10 & 0.128 \\
\hline Human $\rightarrow$ Prawn & 10 & 0.128 \\
\hline Eagle $\rightarrow$ Fish & 11 & 0.127 \\
\hline Human $\rightarrow$ Clam & 12 & 0.123 \\
\hline Human $\rightarrow$ Bear & 13 & 0.111 \\
\hline Human $\rightarrow$ Oyster & 13 & 0.111 \\
\hline Whale $\rightarrow$ Sea lion & 14 & 0.107 \\
\hline Human $\rightarrow$ Dogfish & 15 & 0.104 \\
\hline Eagle $\rightarrow$ Salmon & 16 & 0.103 \\
\hline Seal $\rightarrow$ Fish & 17 & 0.101 \\
\hline
\end{tabular}

\begin{tabular}{|l|c|c|}
\hline $\begin{array}{l}\text { PREDATOR } \rightarrow \text { PREY } \\
\text { RELATIONSHIP }\end{array}$ & $\begin{array}{c}\text { RANK } \\
\text { BY S }\end{array}$ & $\begin{array}{c}\text { SMITH'S } \\
\text { SALIENCE } \\
\text { INDEX (S) }\end{array}$ \\
\hline Killer whale $\rightarrow$ Salmon & 1 & 0.270 \\
\hline Bear $\rightarrow$ Salmon & 2 & 0.267 \\
\hline Wolf $\rightarrow$ Deer & 3 & 0.236 \\
\hline Cougar $\rightarrow$ Deer & 4 & 0.189 \\
\hline Killer whale $\rightarrow$ Sea lion & 5 & 0.138 \\
\hline Sea otter $\rightarrow$ Urchin & 6 & 0.134 \\
\hline Eagle $\rightarrow$ Salmon & 7 & 0.114 \\
\hline Killer whale $\rightarrow$ Seal & 8 & 0.109 \\
\hline Sea otter $\rightarrow$ Crab & 9 & 0.104 \\
\hline Sea lion $\rightarrow$ Fish & 10 & 0.091 \\
\hline Cougar $\rightarrow$ Raccoon & 11 & 0.090 \\
\hline Bird $\rightarrow$ Berries & 12 & 0.085 \\
\hline Whale $\rightarrow$ Plankton & 13 & 0.079 \\
\hline Grey whale $\rightarrow$ Plankton & 13 & 0.079 \\
\hline Wolf $\rightarrow$ Bear & 14 & 0.078 \\
\hline Salmon $\rightarrow$ Herring & 15 & 0.072 \\
\hline Seal $\rightarrow$ Salmon & 16 & 0.067 \\
\hline Wolf $\rightarrow$ Raccoon & 17 & 0.065 \\
\hline Bear $\rightarrow$ Berries & 18 & 0.062 \\
\hline Sea otter $\rightarrow$ Clam & 19 & 0.060 \\
\hline
\end{tabular}

Table A6.3 Most cognitively accessible trophic relationships: females (top 20)

Table A6.4 Most cognitively accessible trophic relationships: males (top 20) 


\begin{tabular}{|l|c|c|}
\hline $\begin{array}{l}\text { PREDATOR } \rightarrow \text { PREY } \\
\text { RELATIONSHIP }\end{array}$ & $\begin{array}{c}\text { RANK } \\
\text { BY S }\end{array}$ & $\begin{array}{c}\text { SMITH'S } \\
\text { SALIENCE } \\
\text { INDEX (S) }\end{array}$ \\
\hline Wolf $\rightarrow$ Deer & 1 & 0.295 \\
\hline Sea otter $\rightarrow$ Urchin & 2 & 0.255 \\
\hline Bear $\rightarrow$ Salmon & 3 & 0.237 \\
\hline Cougar $\rightarrow$ Deer & 4 & 0.229 \\
\hline Sea otter $\rightarrow$ Clam & 5 & 0.206 \\
\hline Killer whale $\rightarrow$ Salmon & 6 & 0.168 \\
\hline Sea otter $\rightarrow$ Crab & 7 & 0.127 \\
\hline Bear $\rightarrow$ Fish & 7 & 0.120 \\
\hline Killer whale $\rightarrow$ Seal & 8 & 0.116 \\
\hline Eagle $\rightarrow$ Salmon & 9 & 0.115 \\
\hline Eagle $\rightarrow$ Fish & 10 & 0.101 \\
\hline Sea otter $\rightarrow$ G. Barnacle & 11 & 0.092 \\
\hline Salmon $\rightarrow$ Herring & 12 & 0.080 \\
\hline Bear $\rightarrow$ Clam & 13 & 0.078 \\
\hline Bear $\rightarrow$ Berries & 14 & 0.075 \\
\hline Killer whale $\rightarrow$ Porpoise & 14 & 0.075 \\
\hline Eagle $\rightarrow$ Sckye. salmon & 15 & 0.073 \\
\hline Bear $\rightarrow$ Blackberry & 16 & 0.070 \\
\hline Wolf $\rightarrow$ Dog & 17 & 0.068 \\
\hline Human $\rightarrow$ Halibut & 18 & 0.067 \\
\hline Bear $\rightarrow$ Sckye. salmon & 19 & 0.065 \\
\hline Sea otter $\rightarrow$ Oyster & 20 & 0.064 \\
\hline Human $\rightarrow$ Deer & 21 & 0.063 \\
\hline Killer whale $\rightarrow$ S.lion & 22 & 0.062 \\
\hline Sea otter $\rightarrow$ Mussel & 23 & 0.060 \\
\hline Sea otter $\rightarrow$ Abalone & 24 & 0.059 \\
\hline Seal $\rightarrow$ Clam & 25 & 0.055 \\
\hline Sea otter $\rightarrow$ Chiton & 26 & 0.054 \\
\hline Cougar $\rightarrow$ Dog & 27 & 0.053 \\
\hline Bear $\rightarrow$ Crab & 27 & 0.053 \\
\hline
\end{tabular}

Table A6.5 Most cognitively accessible trophic relationships: First Nations (top 30)

\begin{tabular}{|l|c|c|}
\hline $\begin{array}{l}\text { PREDATOR } \rightarrow \text { PREY } \\
\text { RELATIONSHIP }\end{array}$ & $\begin{array}{c}\text { RANK } \\
\text { BY S }\end{array}$ & $\begin{array}{c}\text { SMITH'S } \\
\text { SALIENCE } \\
\text { INDEX (S) }\end{array}$ \\
\hline Bear $\rightarrow$ Salmon & 1 & 0.227 \\
\hline Killer whale $\rightarrow$ Sea lion & 2 & 0.196 \\
\hline Killer whale $\rightarrow$ Salmon & 3 & 0.179 \\
\hline Cougar $\rightarrow$ Deer & 4 & 0.160 \\
\hline Sea otter $\rightarrow$ Urchin & 5 & 0.155 \\
\hline Wolf $\rightarrow$ Deer & 6 & 0.151 \\
\hline Sea lion $\rightarrow$ Fish & 7 & 0.148 \\
\hline Cougar $\rightarrow$ Raccoon & 8 & 0.128 \\
\hline Bear $\rightarrow$ Berries & 9 & 0.125 \\
\hline Eagle $\rightarrow$ Salmon & 10 & 0.106 \\
\hline Killer whale $\rightarrow$ Seal & 11 & 0.095 \\
\hline Killer whale $\rightarrow$ Fish & 12 & 0.092 \\
\hline Sea otter $\rightarrow$ Clam & 13 & 0.091 \\
\hline Killer whale $\rightarrow$ Sea otter & 14 & 0.082 \\
\hline Hbck. whale $\rightarrow$ Herring & 14 & 0.082 \\
\hline Starfish $\rightarrow$ Oyster & 14 & 0.082 \\
\hline Whale $\rightarrow$ Plankton & 15 & 0.080 \\
\hline Human $\rightarrow$ Halibut & 17 & 0.078 \\
\hline Eagle $\rightarrow$ Fish & 18 & 0.077 \\
\hline Human $\rightarrow$ Bear & 18 & 0.077 \\
\hline Bear $\rightarrow$ Mussel & 19 & 0.074 \\
\hline Whale $\rightarrow$ Sea lion & 19 & 0.074 \\
\hline Bird $\rightarrow$ Berries & 19 & 0.074 \\
\hline Human $\rightarrow$ Prawn & 20 & 0.073 \\
\hline Human $\rightarrow$ Dogfish & 21 & 0.072 \\
\hline Human $\rightarrow$ Shrimp & 22 & 0.070 \\
\hline Bear $\rightarrow$ Fish & 23 & 0.069 \\
\hline Bear $\rightarrow$ Oyster & 23 & 0.069 \\
\hline Sea otter $\rightarrow$ Crab & 23 & 0.069 \\
\hline Bear $\rightarrow$ Bird & 0.068 \\
\hline
\end{tabular}

Table A6.6 Most cognitively accessible trophic relationships: non-First Nations (top 30) 


\section{Appendix 7}

\section{Logic of group selection}

\section{Patterns of difference in participants' visual depictions of Clayoquot Sound}

We arrived at our choice of a priori demographic subgroups as follows. Our first intimations of systematic differences amongst groups of participants arose with an initial drawing task (see Appendix 7). Namely, as we conducted an increasing number of interviews, we began to observe that males, on average, appeared to depict "the area in which [they] work and/or live" specifically in the form of abstract, overhead maps. Females, on the other hand, tended to depict "the area in which [they] work and/or live" specifically as human-scale landscapes, composed of features such as mountains, water, beaches and trees. Moreover, most of these depictions were largely devoid of people-that is, except in the case of First Nations participants. First Nations' depictions seemed to include people interacting with the environment (e.g., collecting food) much more frequently than did their non-First Nations counterparts' (see Figures 5A.1 to 5A.3).

To test the hypothesis that these observations were indicative of a significant difference amongst these groups, we first coded each participant's visual depiction as a "map" or "scene", respectively, and also noted whether each depiction "contained people" or not. We then used this simple coding scheme to regress participants' visual depictions of their local environment on three demographic variables: gender, First Nations status and-in case we had missed a different underlying driver-also age.

As we hypothesized, males emerged as having much greater odds than women of visually representing their local social-ecological system in the form of an overhead, abstract map, specifically. This was true by a factor of nearly 7:1, $\mathrm{OR}=6.77$ ( $\mathrm{Cl} 1.78-25.72), \mathrm{p}=0.005$. In addition to gender, there was also a sizable difference between First Nations and non-First Nations participants. First Nations emerged as having much greater odds than non-First Nations (more than 6:1) of including people in their visual depictions, $\mathrm{OR}=6.35$ ( $\mathrm{Cl} 1.22-32.91), \mathrm{p}=0.028$.

To be clear, we emphatically are not arguing that these differences suggest biologically or genetically determined variations in cognition. Rather, while we realize highlighting such differences may be provocative, our point is that-regardless of the underlying reason-if and when different "habits of mind" (Medin and Atran 2004) do appear to exist across groups, paying attention to them can serve as a first clue in better revealing and understanding potentially consequential variation in in the way multiple stakeholders are conceptualizing their shared social-ecological system, wittingly or otherwise.

In this case, we suspect that the gender- and cultural differences noted above are largely driven by the different kinds of activities that males versus females, and First Nations versus non-First Nations, tend to engage in most frequently in the Clayoquot Sound region. Commercial and subsistence fishing, which involve coastal navigation, often over large distances, are largely male-dominated activities on the 
west coast of Vancouver Island (WCVI). Thus, men's depictions of their environment may well reflect the habits of mind they form by virtue of repetition during their extended periods of time on the water.

Women in Clayoquot Sound, on the other hand, seem to spend relatively less time navigating long distances in boats. Rather, amongst our female participants, more commonly cited activities involved jobs in the land-based services industry or in near-shore areas. Similarly, while many men listed recreational fishing as a pastime, women participants' preferred recreational activities likewise tended to centre on near-shore or inland areas (e.g., surfing, hiking, beachcombing).

First Nations participants of both genders appear to engage in more subsistence food collection than do their non-First Nations neighbours. Locally harvested salmon and halibut are especially important foods in the diets and cultural economy of regional Nuu-Chah-Nulth First Nations, but so are herring, herring roe (which is collected off kelp or cedar branches lowered into spawning areas), and a wide range of rocky shore invertebrates. Amongst First Nations, it is largely men who traditionally harvest salmon and halibut, while it is women who have historically spent proportionally more time harvesting near-shore shellfish.

Such habits, however, may not entirely account for First Nations' participants' relative penchant for descriptive scenes over maps, nor their significantly greater inclusion of humans in their depictions. There may also be ontological or epistemological differences involved (Ross et al. 2007): for example, a tendency amongst First Nations to think of humans as more directly involved in, or part of, the local social-ecological system, relative to non-First Nations who in the course of our interviews seemed to have more distinct notions of 'nature' as separate from human activity. Regardless of the underlying cause, these significant differences in cognitive style are thought provoking and, we argue, worthy of further consideration in the context of actual multistakeholder consultation or negotiation over shared marine resources in the region. 
Figure A7.1: Sample depiction of local ambit-non-First Nations male

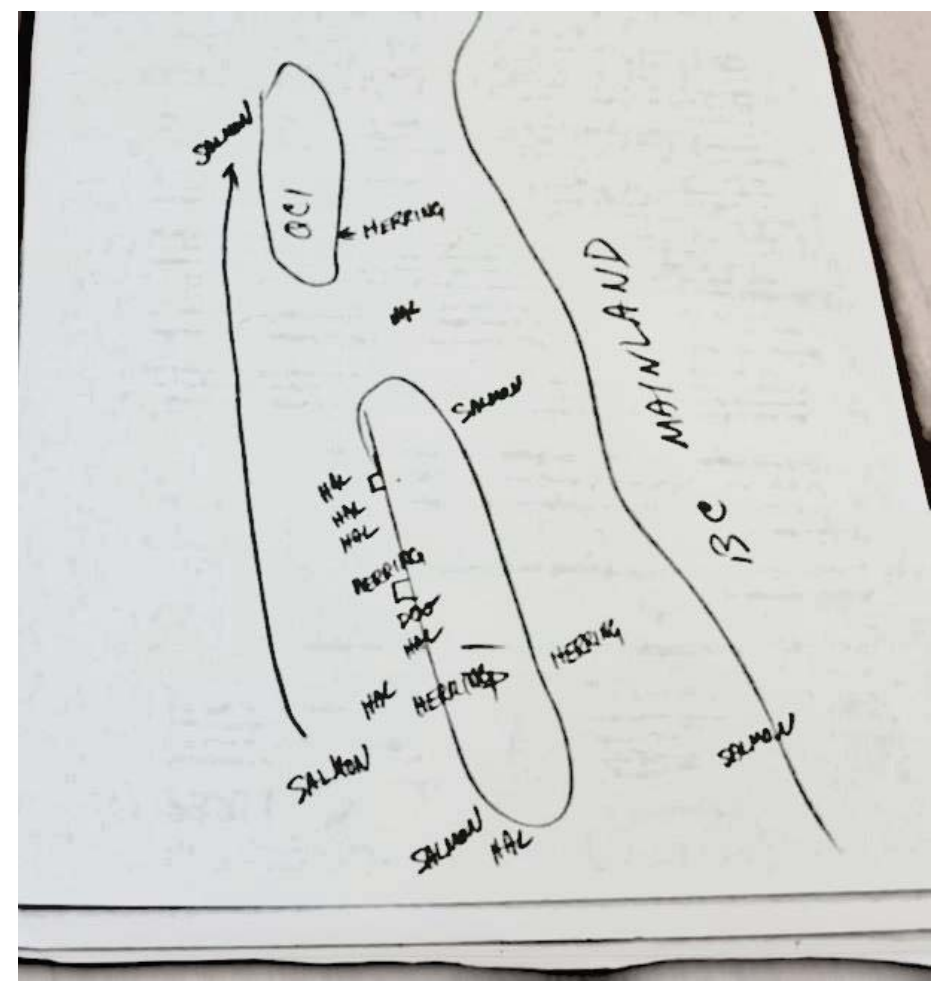

This image is an example of one non-First Nation male participant's response to the instruction "please depict the area in which you work and/or live." Note that this participant chose an overhead map-style depiction, at a fairly large scale, with an emphasis on fishing activities. Men exhibited, on average, nearly seven (6.77) times greater odds of intuitively choosing this map-based style of depiction than did women $(p=0.005)$. 
Figure A7.2: Sample depiction of local ambit-non-First Nations female

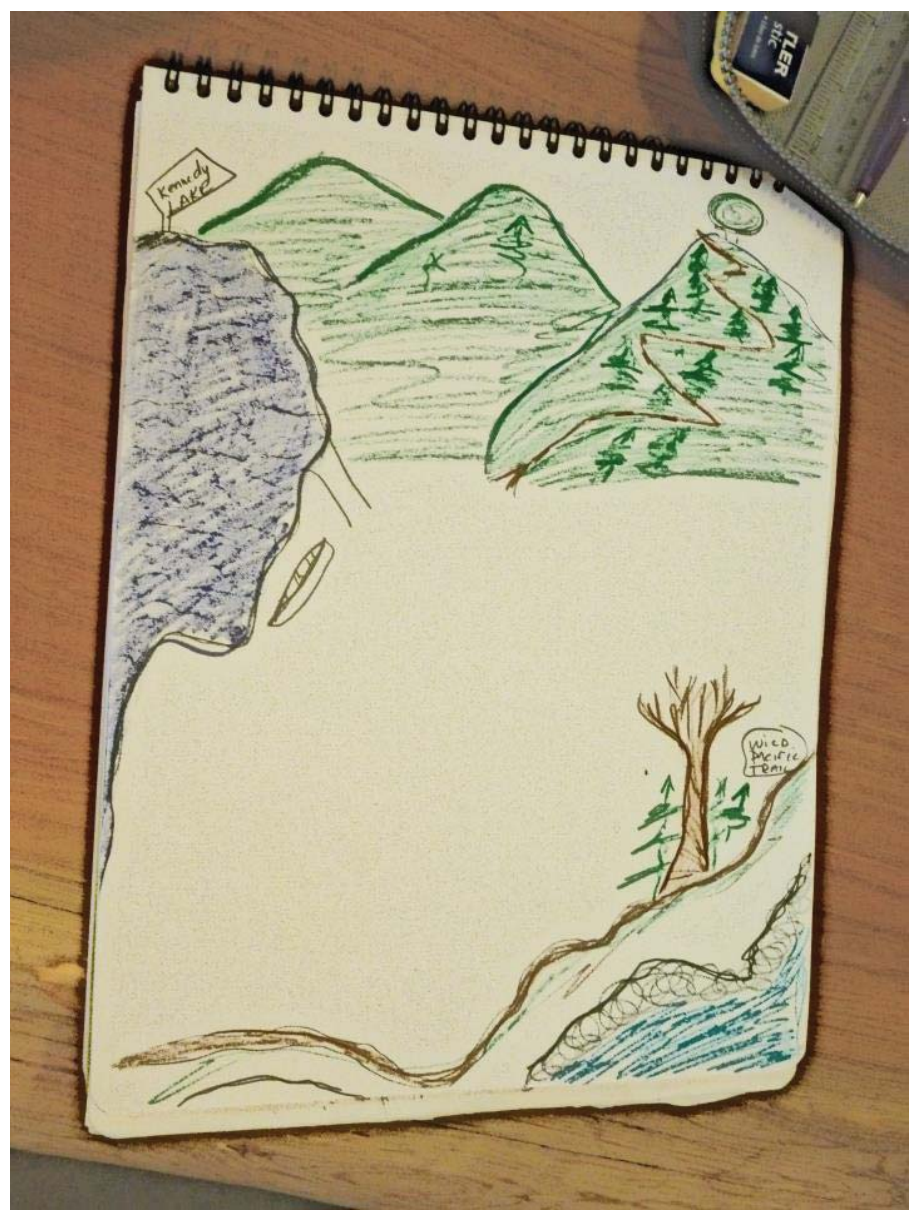

This image is an example of one non-First Nation female participant's response to the instruction "please depict the area in which you work and/or live." Note that this participant chose a natural scene, at a relatively high-resolution scale, depicting both land and coastline, with no humans in the image. Women exhibited, on average, nearly seven (6.77) times greater odds of intuitively choosing a scenebased style of depiction (such as this, above) than did men $(p=0.005)$. 
Figure A7.3: Sample depiction of local ambit: First Nations male

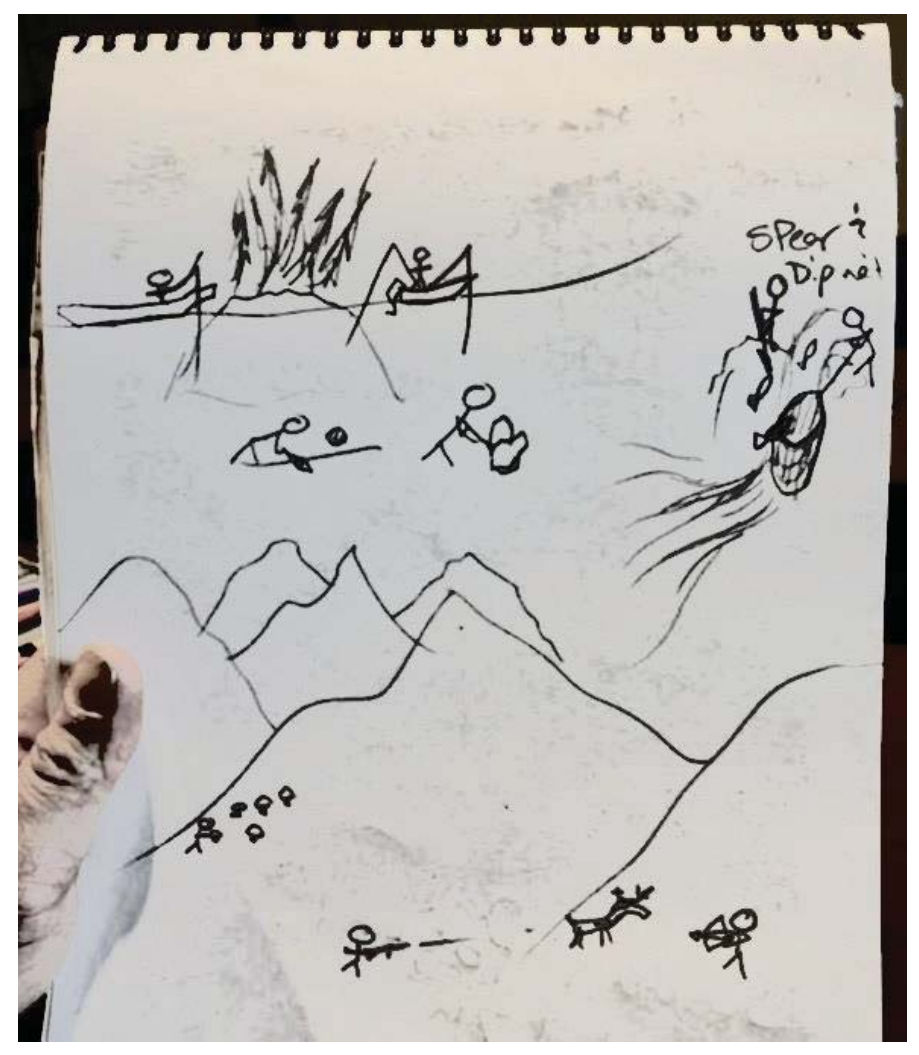

This image is an example of one First Nations male participant's response to the instruction "please depict the area in which you work and/or live." Note that this participant chose a series of outdoors scene, at a relatively high-resolution scale, depicting both land and water, with many humans in the image, each of which is conducting a different form of wild food collection. First Nations participants had, on average, over six (6.35) times higher odds of intuitively including humans in their depiction than did non-First Nations $(p=0.028)$. 